\title{
Cortical somatosensory reorganization in children with spastic cerebral palsy: a multimodal neuroimaging study
}

\author{
Christos Papadelis ${ }^{1,2}{ }^{+}$, Banu Ahtam ${ }^{1,2+}{ }^{+}$, Maria Nazarova ${ }^{3,4}$, Donna Nimec $^{5}$, Brian Snyder ${ }^{5}$, \\ Patricia Ellen Grant ${ }^{1,2,6}$ and Yoshio Okada ${ }^{1,2}$ \\ ${ }^{1}$ Fetal-Neonatal Neuroimaging and Developmental Science Center, Boston Children's Hospital, Harvard Medical School, Boston, MA, USA \\ ${ }^{2}$ Division of Newborn Medicine, Department of Medicine, Boston Children's Hospital, Harvard Medical School, Boston, MA, USA \\ ${ }^{3}$ Department of Neurorehabilitation and Physiotherapy, Research Center of Neurology, Moscow, Russia \\ ${ }^{4}$ Centre for Cognition and Decision Making, Faculty of Psychology, Higher School of Economics, Moscow, Russia \\ ${ }^{5}$ Department of Orthopaedic Surgery, Boston Children's Hospital, Harvard Medical School, Boston, MA, USA \\ ${ }^{6}$ Department of Radiology, Boston Children's Hospital, Harvard Medical School, Boston, MA, USA
}

\section{Edited by:}

Hubert Preissl, University of Tübingen, Germany

\section{Reviewed by:}

Giancarlo Zito, San Giovanni Calibita

Fatebenefratelli Hospital, Italy

Douglas Rose, Cincinnati Children's

Hospital Medical Center and

University of Cincinnati, USA

\section{*Correspondence:}

Christos Papadelis, Boston Children's Hospital, Harvard Medical School,

BabyMEG Facility, 9 Hope Avenue, Waltham, MA 02453, USA

e-mail: christos.papadelis@childrens. harvard.edu

${ }^{\dagger}$ Christos Papadelis and Banu Ahtam are the co-first authors.
Although cerebral palsy (CP) is among the most common causes of physical disability in early childhood, we know little about the functional and structural changes of this disorder in the developing brain. Here, we investigated with three different neuroimaging modalities [magnetoencephalography (MEG), diffusion tensor imaging (DTI), and resting-state fMRI] whether spastic $\mathrm{CP}$ is associated with functional and anatomical abnormalities in the sensorimotor network. Ten children participated in the study: four with diplegic CP (DCP), three with hemiplegic CP (HCP), and three typically developing (TD) children. Somatosensory (SS)-evoked fields (SEFs) were recorded in response to pneumatic stimuli applied to digits D1, D3, and D5 of both hands. Several parameters of water diffusion were calculated from DTI between the thalamus and the pre-central and post-central gyri in both hemispheres. The sensorimotor resting-state networks (RSNs) were examined by using an independent component analysis method. Tactile stimulation of the fingers elicited the first prominent cortical response at $\sim 50 \mathrm{~ms}$, in all except one child, localized over the primary SS cortex (S1). In five CP children, abnormal somatotopic organization was observed in the affected (or more affected) hemisphere. Euclidean distances were markedly different between the two hemispheres in the HCP children, and between DCP and TD children for both hemispheres. DTI analysis revealed decreased fractional anisotropy and increased apparent diffusion coefficient for the thalamocortical pathways in the more affected compared to less affected hemisphere in CP children. Resting-state functional MRI results indicated absent and/or abnormal sensorimotor RSNs for children with HCP and DCP consistent with the severity and location of their lesions. Our findings suggest an abnormal SS processing mechanism in the sensorimotor network of children with CP possibly as a result of diminished thalamocortical projections.

Keywords: cerebral palsy, cortical reorganization, somatosensory processing, magnetoencephalography, MR tractography, resting-state functional MRI

\section{INTRODUCTION}

Cerebral palsy (CP) is a well-recognized group of motor and postural neurodevelopmental disorders beginning in early childhood and persisting through the lifespan. CP causes serious motor impairments often accompanied by disturbances of sensation and perception (Bax et al., 2005) and is the most common cause of physical disability in early childhood (Krageloh-Mann and Cans, 2009). Almost 3.6 out of every 1000 children born in the US suffer from $\mathrm{CP}$ and the prevalence of the disorder is on the rise worldwide (Yeargin-Allsopp et al., 2008). CP involves axonal and neuronal loss in cerebral white and gray matter, reduction in thalamocortical connections (Hoon et al., 2002, 2009; Thomas et al., 2005; Nagae et al., 2007), and comparable loss in subcortical structures (Volpe, 2009). Depending on the location, extent, and timing of the insult, clinical symptoms vary largely.
Spastic CP (SCP) is the most common form of CP that frequently alters the normal development of the somatosensory (SS) system. SCP is usually presented with increased muscle tone, hyperreflexia, and persistence of primitive reflexes (Tomlin, 1995). Aside from motor and postural impairments, children with SCP frequently experience sensory deficits such as altered tactile, proprioceptive, kinesthetic, and pain awareness (Van Heest et al., 1993; Cooper et al., 1995; Krumlinde-Sundholm and Eliasson, 2002; Sanger and Kukke, 2007; Wingert et al., 2009; Riquelme et al., 2011). Recent functional neuroimaging studies have reported altered SS processing in SCP individuals as measured by SS-evoked potentials or fields in terms of amplitude, morphology, frequency power, or somatotopy (Riquelme and Montoya, 2010; Kurz and Wilson, 2011; Teflioudi et al., 2011; Guo et al., 2012; Nevalainen et al., 2012; Pihko et al., 2014; Riquelme et al., 2014). In a recent 
magnetoencephalography (MEG) study, Pihko et al. (2014) found that suppression and rebound of alpha and beta cortical activity to contralateral stimulation were smaller in the lesioned compared to the intact hemisphere in hemiplegic $\mathrm{CP}$ ( $\mathrm{HCP})$ children, while they did not find any difference between the hemispheres of typically developing (TD) children. A functional MRI study, which investigated tactile shape and grating discrimination, found decreased cortical activity in the parietal and frontal cortical SS regions of SCP compared to TD children (Wingert et al., 2010).

Anatomical neuroimaging studies using diffusion tensor imaging (DTI) have provided evidence of significant alterations in white matter fibers connecting to sensory cortex. These studies suggest that $\mathrm{CP}$ injuries might be reflective of disruption of sensory as well as motor connections. They also provide evidence of sensory and motor pathway involvement for the motor weakness in $\mathrm{CP}$ patients by showing that DTI measures reflect the degree of motor deficits (Hoon et al., 2002, 2009; Thomas et al., 2005; Trivedi et al., 2010). Two DTI studies observed more severe damage in the posterior white matter fibers connecting the thalamus to the sensory cortex than in the descending corticospinal tracts in children with periventricular leukomalacia (PVL) (Hoon et al., 2002) and SCP (Nagae et al., 2007), despite a history and clinical presentation consistent with motor tracts. Compared to TD children, children with $\mathrm{CP}$ have been reported to have decreased fractional anisotropy (FA) and increased apparent diffusion coefficient (ADC) values for the motor and sensory tracks (Trivedi et al., 2010); for the affected side of the corticospinal tract (Son et al., 2007; Yoshida et al., 2010); and for the tracks on the side ipsilateral to the periventricular lesion in corticospinal tract, corticobulbar tract, and superior thalamic radiation (Thomas et al., 2005).

Fundamental understanding of sensory function in $\mathrm{CP}$ children is extremely important, since SS input is an essential component of motor function, control, and development. Tactile inputs are used to localize and characterize the various qualities of touch, while cutaneous inputs contribute to proprioceptive information for coordinated motor actions (Dijkerman and de Haan, 2007). Both tactile and cutaneous inputs play an important role in the proprioceptive feedback for motor planning and execution (Wingert et al., 2008). Sensibility deficiencies have been correlated with diminished dexterity in the affected hand of CP children with spastic hemiplegia (Van Heest et al., 1993). It has been suggested that in $\mathrm{CP}$ there is a loss of coordinated messages from SS to motor areas (Burton et al., 2009), which may lead to deficits in motor coordination (Bax et al., 2005), fine and gross motor function (Himmelmann et al., 2006), and motor control (Ostensjo et al., 2004; Fowler and Goldberg, 2009). Deficits in the processing of SS information may also partially explain the tactile or motor deficits observed in this population (Wingert et al., 2008; Burton et al., 2009).

Somatosensory inputs are also important for the development of motor system; early learning in infants is driven largely by SS inputs. Moreover, the ability to process and utilize sensory information for motor planning control develops through the childhood (Riquelme and Montoya, 2010; Gordon et al., 2013). Thus, defective tactile/cutaneous feedback may worsen motor planning and performance (Gordon et al., 2013). CP children with even mild motor impairments have demonstrated more variable and redundant fingertip forces while adjusting to objects compared to TD, presumably, at least partly, due to imperfect proprioceptive feedback (Gordon et al., 2013). It is possible that there is also an opposite mechanism: deficits of spontaneous movements in $\mathrm{CP}$ infants (Prechtl, 1997; Prechtl et al., 1997; Hadders-Algra, 2004; Einspieler and Prechtl, 2005) may account for musculoskeletal tissue changes and thus contribute to aberrant sensory inputs to the brain (Coq et al., 2008). Understanding the pathophysiology mechanisms underlying the sensory impairments, specifically tactile, in $\mathrm{CP}$ children is essential to the design of effective therapeutic interventions.

In the present study, we investigated with three different neuroimaging modalities [pediatric MEG, DTI, and resting-state functional MRI (rs-fMRI)] whether SCP is associated with functional and anatomical abnormalities in the sensorimotor network. To our best knowledge, this is the first study that combines findings from multiple neuroimaging techniques for examining the anatomical and functional integrity of the SS and motor systems in children with SCP. Somatosensory-evoked fields (SEFs) in response to pneumatic stimulation of the fingertips were recorded with pediatric MEG and the underlying generators were localized by using minimum norm estimates (MNE) (Hamalainen and Ilmoniemi, 1994). MEG was used because it is able to elucidate the dynamic spatiotemporal characteristics of SS cortical activation (Lin et al., 2006a) with its excellent temporal and good spatial accuracy (Hämäläinen et al., 1993; Papadelis et al., 2009). So far, there are very few MEG studies reporting functional abnormalities in the SS cortex of CP children (Kurz and Wilson, 2011; Nevalainen et al., 2012; Pihko et al., 2014). With MEG, we tested our hypotheses that the SEFs will be abnormal and the primary cortical SS representation areas will present an altered somatotopy in the affected (or more affected) compared to the intact (or less affected) hemisphere of $\mathrm{CP}$ children, and compared to both hemispheres of TD children. The integrity of the thalamocortical tracts from thalamus to the pre-central and post-central gyri was examined in the same participants by using DTI. We expected a disruption in the thalamocortical tracts projecting from thalamus to the pre-central and post-central gyri in children with $\mathrm{CP}$. The integrity of the sensorimotor resting-state network (RSN) functional architecture was also assessed by measuring spontaneous low-frequency fluctuations $(<0.1 \mathrm{~Hz})$ in the blood oxygen leveldependent (BOLD) signal by using an independent component analysis (ICA) method. We hypothesized that the cortical networks linked to areas within the SS and motor cortices would be either absent or at least abnormal in children with CP.

\section{MATERIALS AND METHODS PARTICIPANTS}

Ten right-handed children participated in the study: four children with diplegic CP (DCP) (two males and two females, mean age: 11.8 years, SD 5.4 years), three children with HCP (two males and one female, mean age: 12.6 years, SD 5.8 years), and three age-matched TD children (one male and two females, mean age: 11.8 years, SD 5.2 years). CP patients were recruited from the Cerebral Palsy Clinic at Boston Children's Hospital (BCH), Harvard Medical School, according to the following inclusion criteria: (1) evaluation by a pediatric neurologist and diagnosis of DCP 
or HCP, (2) absence of any genetic syndrome diagnosis, (3) no history of trauma or brain operation, and (4) classified as highfunctioning in level I or II at the Gross Motor Function Classification System (GMFCS) (Palisano et al., 1997). TD comparison children had no history of neurological disorder or brain injury. None of the participants were under psychoactive or myorelaxant medications during the experiment. Table 1 presents the demographic, clinical, and conventional MRI data for all participants. All participants were free of metallic objects or implant devices, and cooperative to understand and follow simple instructions. This study was approved by the local institutional review board and informed written consent was obtained from the parents of all participants.

\section{MEG RECORDINGS}

SEFs were elicited by tactile stimulation of the D1, D3, and D5 (thumb, middle, and little finger) of both hands. The stimuli were delivered through thin elastic membranes (with a diameter of $1 \mathrm{~cm}$ ), surrounded by a plastic outer shell, which were attached to the distal, volar parts of the three digits. Membranes were inflated by an air pressure pulse through a rigid plastic tube (Somatosensory Stimulus Generator, 4D NeuroImaging Inc., San Diego, CA, USA) tapping gently the skin at the tip of each digit. The pressure of the tactile stimulator rose to 0.10 bar overpressure in $10 \mathrm{~ms}$. Tactile stimuli were delivered to the three digits in a pseudorandom order with an interstimulus interval (ISI) of $1.5 \pm 0.5 \mathrm{~s}$. Each finger received in total 180 stimuli in three runs. Each run lasted $\sim 6 \mathrm{~min}$. The measurements were carried out in the BabyMEG Facility at BCH (Waltham, MA, USA). MEG signals were recorded inside a single-layer magnetically shielded room (MSR) (Imedco, Hägendorf, Switzerland) with a 76-axial gradiometers device (BabySQUID Tristan Technologies, Inc., San Diego, CA, USA). The sensor array in BabySQUID consists of 74 active gradiometers ( $10 \mathrm{~mm}$ pickup coil diameter, $30 \mathrm{~mm}$ baseline, and 12-14 mm coil center-to-coil center spacing) with a gap between each pickup coil and scalp of $\sim 7-10 \mathrm{~mm}$. The sensor array is ellipsoidal in shape with a radius of curvature of $7.5 \mathrm{~cm}$ along the coronal section, $10 \mathrm{~cm}$ along the sagittal section (Okada et al., 2006), and a depth of $\sim 2 \mathrm{~cm}$ resulting in a coverage area of $\sim 265 \mathrm{~cm}^{2}$. The recording signal bandwidth was $0-341.33 \mathrm{~Hz}$ with a sampling rate of $1024 \mathrm{~Hz}$. Vertical and horizontal electrooculograms (EOGs) and electrocardiogram (ECG) were simultaneously recorded using bipolar electrodes.

During the recordings, the children were comfortably lying down on the bed with the hemisphere contralateral to the stimulated hand placed on the headrest in such a way that the sensor array was covering the scalp area above the pre-central and post-central gyri. We focused our recordings on the contralateral hemisphere because previous evidence in $\mathrm{CP}$ children present that the SS representation remains in the hemisphere contralateral to the affected hand in contrast to the motor system (Gerloff et al., 2006; Staudt et al., 2006; Wilke and Staudt, 2009). The children kept their head still during the recordings with eyes open gazing at a fixation point on the wall. Prior to each run an anatomical coordinate system was defined by digitizing 15 points marked on the participant's face. More details about the followed coregistration procedure can be found in Papadelis et al. (2013).

\section{MRI/DTI ACOUISITION}

MRI data were collected with a 3-T Siemens Tim Trio MR scanner at BCH. The data from one child with DCP (DCP4) were excluded due to excessive motion artifact. The scans were performed without sedation or medication, while the children were awake. The imaging protocol consisted of structural and diffusion-weighted sequences. The structural sequence was a T1-weighted high-resolution magnetization-prepared rapidacquisition gradient-echo (MPRAGE) acquisition, which used volumetric echo-planar imaging (EPI) navigators for real time motion correction [voxel size $(\mathrm{mm})=1 \times 1 \times 1$; field of view $(\mathrm{FOV})=19.2-22.0 \mathrm{~cm}$; echo time $(\mathrm{TE})=1.74 \mathrm{~ms}$; repetition time $(\mathrm{TR})=2520 \mathrm{~ms}$; flip angle $=7^{\circ}$. The diffusion sequence (prescribed axially) used echo-planar (EP) readouts [voxel

Table 1 | Demographics and anatomical information.

\begin{tabular}{|c|c|c|c|c|c|}
\hline Participant & Age & Gender & GMFCS & Affected side & Brain MRI \\
\hline TD 1 & 12 years & $\mathrm{F}$ & N/A & $\mathrm{N} / \mathrm{A}$ & Normal \\
\hline TD 2 & 17 years & $\mathrm{F}$ & N/A & $N / A$ & Normal \\
\hline TD 3 & 6.5 years & $\mathrm{M}$ & N/A & $\mathrm{N} / \mathrm{A}$ & Normal \\
\hline HCP 1 & 17 years & $\mathrm{M}$ & 2 & Right & $\begin{array}{l}\text { Chronic encephalomalacia due to left middle } \\
\text { cerebral artery infarct }\end{array}$ \\
\hline HCP 2 & 15 years & $\mathrm{F}$ & 2 & Left & Perinatal right periventricular white matter injury \\
\hline HCP 3 & 6 years & M & 1 & Right & $\begin{array}{l}\text { Subtle gliosis in the left central semiovale extending } \\
\text { to the corona radiata }\end{array}$ \\
\hline DCP 1 & 12 years 9 months & $\mathrm{F}$ & 2 & Both (right more) & Slight asymmetry of the left lateral ventricle \\
\hline DCP 2 & 12 years & $\mathrm{F}$ & 2 & Both (right more) & Bilateral periventricular white matter injury \\
\hline DCP 3 & 16 years & M & 1 & Both (left more) & No gross MRI abnormality \\
\hline DCP 4 & 4 years 9 months & M & 2 & Both (right more) & Periventricular leucomalacia (PVL)a \\
\hline
\end{tabular}

${ }^{a} \mathrm{MRI}$ data were excluded due to excessive motion artifact. PVL diagnosis was given based on previous acquired MRI not available to this study. 
size $(\mathrm{mm})=2.0 \times 2.0 \times 2.0 ; \quad F O V=11-12.8 \mathrm{~cm} ; \quad \mathrm{TE}=88 \mathrm{~ms}$; $\mathrm{TR}=8320-10934 \mathrm{~ms}$; flip angle $=90^{\circ} ; 30$ gradient diffusion directions at $b=1000 \mathrm{~s} / \mathrm{mm}^{2} ; 10$ acquisitions with $\left.b=0 \mathrm{~s} / \mathrm{mm}^{2}\right]$.

\section{rs-fMRI RECORDINGS}

The rs-fMRI data were collected with the same scanner at $\mathrm{BCH}$ after collecting the T1 and DTI sequences. The data from one child with DCP (DCP4) were excluded due to excessive motion artifact. The participants were asked to stay awake and keep their eyes open. The rs-fMRI sequence used EPI readouts with isotropic voxel sizes of $3 \mathrm{~mm} \times 3 \mathrm{~mm} \times 3 \mathrm{~mm}$, whole brain coverage, $\mathrm{TE}=30 \mathrm{~ms}$, $\mathrm{TR}=3 \mathrm{~s}, 47$ axial slices, and 160 time series volumes.

\section{ANALYSIS OF EVENT-RELATED MAGNETIC FIELDS}

MEG data analysis was performed by using Brainstorm (Tadel et al., 2011), which is documented and freely available for download online under the GNU general public license ${ }^{1}$. The recorded MEG signals were visually inspected for possible artifacts and filtered offline in the frequency band of $1-100 \mathrm{~Hz}$. Trials contaminated by prominent eye movements, blinks, or muscular activity were rejected. The remaining trials were then averaged (approximately 60 trials for each stimulation site) for each separate run from -100 to $400 \mathrm{~ms}$ relative to the stimulus onset. Grand averages across all runs were estimated per stimulation site for each participant. There was no significant difference in the number of useful trials between stimulation sites or groups $(p>0.05)$. The peak latency of the first cortical response was visually determined from the grand average butterfly plot for each site and each participant.

\section{SOURCE LOCALIZATION ANALYSIS}

An MRI-derived surface model of each participant's brain was initially estimated from the T1-weighted structural volumetric images by using FreeSurfer ${ }^{2}$. For the participant with no MRI, a template MRI from an age-matched child was used. The geometry of the gray-white matter surface was derived with an automatic segmentation algorithm to yield a triangulated model with approximately 270,000 vertices (Dale et al., 1999; Fischl et al., 1999, 2001). For computational purposes, the source space was obtained by decimating the original triangulation to a subset of vertices ( $\sim 15,000$ vertices $)$ with an average of $5 \mathrm{~mm}$ distance between nearest dipoles. Each vertex represents a given source space point defined as an equilateral triangle in the tessellation of the cortical surface. To compute the forward model, the overlapping-sphere method was used for each participant that fits one local sphere for each sensor (Huang et al., 1999) and then derives the strength of a set of current dipoles located at the cortical surface. The distributed source model of the MEG signals was then estimated by using the MNE (Hamalainen and Ilmoniemi, 1994). MNE was selected because it does not require explicit a priori assumptions about the nature or number of source currents (Hamalainen and Ilmoniemi, 1994). It has been suggested to be the preferred method when analyzing multi-source SS-evoked activations compared to other inverse approaches (Lin et al., 2006a). Using distributed source analysis, the activation at each vertex was estimated at the peak of

\footnotetext{
${ }^{1}$ http://neuroimage.usc.edu/brainstorm

${ }^{2}$ http://surfer.nmr.mgh.harvard.edu/
}

the first cortical response in the evoked fields obtained for each stimulus site and subject. During the computation of the inverse solution, we followed a previously described data analysis strategy (Hsiao et al., 2013): (i) the source orientations were constrained to be perpendicular to the cortical surface; (ii) a depth weighting algorithm was used to compensate for any bias affecting the superficial sources calculation (Lin et al., 2006b); and (iii) a regularization parameter, $\lambda^{2}=0.33$ was used to minimize numerical instability to reduce the sensitivity of the MNE to noise and to effectively obtain a spatially smoothed solution (Hamalainen and Ilmoniemi, 1994). The noise covariance matrix was computed from empty MSR recordings, which always preceded the actual recording sessions. MNE estimates were averaged across different runs after being coregistered in the same coordinate system. Regions of interest (ROIs) for the MEG analysis were selected as the global maxima of cortical activity at the peak of the first cortical response after the stimuli onset. These ROIs will be referred to from now on as MEG-defined ROIs. Each MEG-defined ROI consisted of 10 neighboring vertices surrounding the vertex with the global maximum activation at the peak of the first cortical response after the stimuli onset. The average size of MEG-defined ROIs was $0.792 \pm 0.169 \mathrm{~mm}$. The mean distance of the vertices defining the ROI from the central vertices was $3.4 \pm 1.47 \mathrm{~mm}$. Then, the exact location of each MEG-defined ROI was displayed on the anatomical MRI of each individual.

\section{MRI-DEFINED ROIs}

T1 and diffusion data were processed with Connectome Mapper (CMP) (Daducci et al., 2012) pipeline, which includes the use of several neuroimaging tools, such as the FreeSurfer ${ }^{2}$. The CMP-generated file ROI_HR_th.nii.gz was used to create volume files for thalamus, pre-central gyrus, and post-central gyrus using the mri_binarize command of FreeSurfer. All three volumes were checked and manually edited in FreeView to ensure correct segmentation. The three volumes and the $\mathrm{T} 1$ image were coregistered with the b0 image using 3D Slicer software ${ }^{3}$. The transformed volumes were then imported in TrackVis software ${ }^{4}$ as ROIs. They will be referred to from now on as MRI-defined ROIs.

\section{FIBER TRACTOGRAPHY}

Diffusion data were processed with Diffusion Toolkit ${ }^{5}$ using HARDI/Q-Ball imaging model and second order Runge Kutta propagation algorithm with an angle threshold of $45^{\circ}$ and no FA threshold. Fiber tractography was performed with TrackVis software to create fiber tracks that pass through thalamus and post-central gyrus as well as thalamus and pre-central gyrus. Some spurious connections ( $<3 \%$ ) were manually removed using TrackVis. Mean number of fibers as well as mean scalar measures of FA, ADC, axial diffusivity (AD), and radial diffusivity (RD) were derived for each fiber track. Data were analyzed separately for the two hemispheres identified as affected (or more affected) and intact (or less affected) hemispheres in CP children, and for right and left hemispheres for TD children.

\footnotetext{
${ }^{3}$ http://www.slicer.org

${ }^{4}$ http://www.trackvis.org

${ }^{5}$ http://www.trackvis.org/dtk/
} 


\section{RESTING-STATE fMRI}

For the rs-fMRI analysis, we followed the same methodology as in Dehaes et al. (2013). Anatomical T1-weighted DICOM images were converted into a 3D NIFTI file format using the mri_convert command of FreeSurfer ${ }^{2}$. Then, the T1 image of each participant was manually oriented into Talairach space using the anterior commissure (AC) and posterior commissure (PC) landmarks using FreeView. Brain extraction tool (BET) was used to remove the non-brain tissue from the T1 image (Smith, 2002). Artifact detection for rs-fMRI raw data was performed using Artifact Rejection Tools (ART) ${ }^{6}$. All participants, except for one child with HCP who had three volumes affected by motion, were free of motion artifacts. After the removal of contaminated volumes, the remaining time series were converted into a 4D NIFTI volume file using mri_convert command of FreeSurfer. Slice timing correction was applied to the rs-fMRI data to correct for sampling offsets using the slicetimer command of FSL. The regression of motion signal was achieved using the mcflirt command of FSL (Jenkinson et al., 2002). Then, the rs-fMRI data were registered in the brain extracted structural T1-weighted image using the FSL's intensitybased affine registration tool, flirt ${ }^{7}$ (Jenkinson et al., 2002). Before the statistical analysis, signal from cerebrospinal fluid (CSF) and white matter was regressed out.

For the statistical analysis, we used the Multivariate Exploratory Linear Optimized Decomposition into Independent Components (melodic) command of the FSL software, which allowed us to decompose the 4D rs-fMRI data sets into its spatial and temporal components with the use of ICA, which can be used to identify distinct RSNs (Beckmann and Smith, 2004). Number of

${ }^{6} \mathrm{http}: / /$ www.nitrc.org/projects/artifact_detect ${ }^{7}$ www.fmrib.ox.ac.uk/fsl components was not specified a priori. In this study, we have identified a sensorimotor RSN of all participants.

\section{RESULTS}

\section{CLINICAL DATA AND MRI}

Table 1 describes the type of lesions in the HCP and DCP children. No gross MRI abnormality was observed in one DCP child. All CP participants had visible asymmetry on the FA maps on the corticospinal tracts. HCP1 had severe spastic paresis of the upper limb and moderately impaired tactile sensory function and proprioception. HCP3, DCP1, and DCP4 had mild paresis of the hand and normal sensory function. The rest of CP participants had no evidence of motor or sensory impairment.

\section{DISTRIBUTED SOURCE ANALYSIS}

Figure 1 shows the superimposed (butterfly) plots of the SEFs for a TD, an HCP, and a DCP child evoked by the tactile stimulation of right D1 and left D1, respectively. A weak reflection at $\sim 30 \mathrm{~ms}$ was observed as the first cortical response after the stimulus onset, but only in four participants (two TD, one HCP, and one DCP) and only after few digits' stimulation: TD1: left hand D1 at $28 \mathrm{~ms}, \mathrm{D} 3$ at $27 \mathrm{~ms}$; TD2: right hand D1 at $32 \mathrm{~ms}$, left hand D1 at $28 \mathrm{~ms}$ and D5 at $27 \mathrm{~ms}$; HCP1: left hand D1 at $28 \mathrm{~ms}, \mathrm{D} 3$ at $30 \mathrm{~ms}, \mathrm{D} 5$ at $31 \mathrm{~ms}$; DCP2: right hand D1 at $29 \mathrm{~ms}$. The most prominent early deflection evoked by the tactile stimulation of D1, D3, and D5 of both hands was observed at around 40-50 ms (M50) in all TD (mean latency \pm SD for D1: $42.62 \pm 3.55 \mathrm{~ms}$, D3: $43.82 \pm 3.88 \mathrm{~ms}$, and D5: $44.24 \pm 4.6 \mathrm{~ms}$ ) and all DCP children (mean latency \pm SD for D1: $43.36 \pm 4.1 \mathrm{~ms}, \mathrm{D} 3: 45.03 \pm 4.5 \mathrm{~ms}$, and D5: $45.06 \pm 2.0 \mathrm{~ms})$. For the lowest functioning HCP child (HCP1), the M50 was absent in the SEFs elicited from the stimulation of the D1, D3, and D5 of the paretic hand (see Figure 1 for D1), while components at later latencies were present. Tactile stimulation of the non-paretic

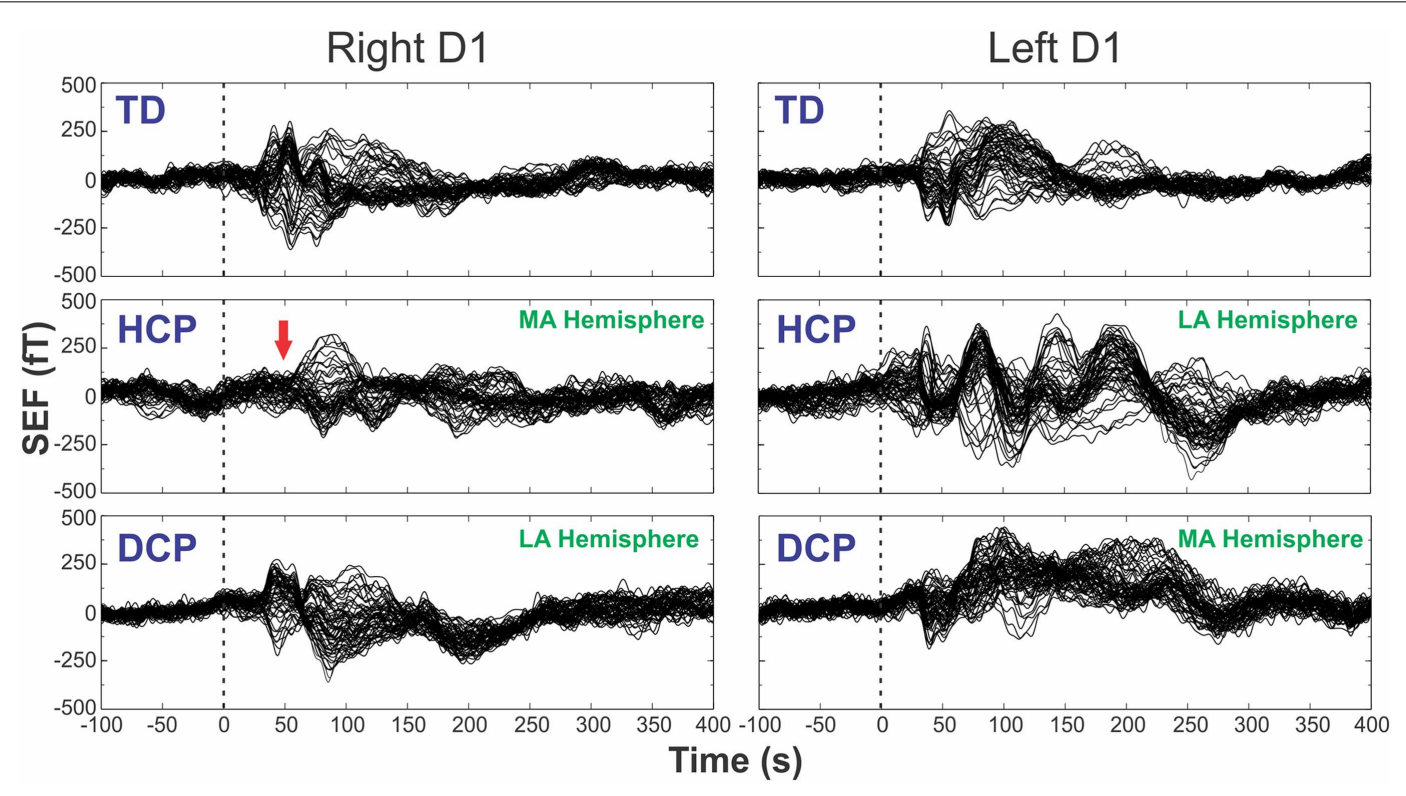

FIGURE 1 | SEFs evoked by the tactile stimulation of the right (left panels) and left (right panels) D1 from TD2, HCP1, and DCP3 children from -100 to $400 \mathrm{~ms}$ after the stimulus onset. Red arrow indicates the absence of the first cortical response in the HCP1 participant. 
hand in the HCP children indicated altered SEFs in the later latencies. The M50 was present in the other two HCP children in both hemispheres. Isofield maps determined that the equivalent current dipoles (ECDs) for the components labeled as M30 presented an anterior ECD direction, while ECDs for the M50 components presented a posterior ECD direction.

Figure 2 shows the butterfly plots of the SEFs for TD1 (upper panel) and DCP2 (lower panel) child accompanied by the corresponding MNE solutions at the peak of M50. At this latency, distributed activations were also observed in the post-central and pre-central gyri and the parietal lobe contralateral to the stimuli for all TD and DCP children. Figure 3 presents the M50 current sources (defined by maximal activities in the MNE maps) at the contralateral primary SS cortex $(\mathrm{S} 1)$ for the stimulation of D1, D3, and D5 (both hands) for three representative participants from each group. Source analysis focused on the M50 that provided sufficient signal-to-noise (SNR) for reliable localization of the underlying generator. The M50 sources contralateral to the stimuli were located in the S1 area of the hand following a somatotopic order for both hands in all TD children (see Figure 3-upper panel for TD1), in all HCP children for the non-paretic hand (see Figure 3 - middle panel - third column for HCP1), and in two DCP for the less paretic hand (see Figure 3 - lower panel third column for DCP2): D5 medial and superior to D3, and D3 medial and superior to D1. An altered somatotopy was observed for the more paretic hand in two DCP children, and in the nonparetic hand in one HCP child (HCP2): D3 was located more posterior and inferior to D1 (Figure 3 - lower panel - second column for DCP2). Euclidean distances were markedly different among evoked activities in S1 between the TD group [mean distance \pm SD (both hemispheres): for D1-D3: $7.38 \pm 1.95 \mathrm{~mm}$, for D3-D5: $6.89 \pm 5.18 \mathrm{~mm}$, for D1-D5: $8.18 \pm 5.54 \mathrm{~mm}$ ] and the
HCP group for the more affected hand (mean distance \pm SD: for D1-D3: $0.0 \pm 0.00 \mathrm{~mm}$, for D3-D5: $0.00 \pm 0.00 \mathrm{~mm}$, for D1-D5: $0.00 \pm 0.00 \mathrm{~mm}$ ) and the less affected hand (mean distance \pm SD: for D1-D3: $12.95 \pm 5.05 \mathrm{~mm}$, for D3-D5: $10.58 \pm 11.77 \mathrm{~mm}$, for D1-D5: $21.47 \pm 9.44 \mathrm{~mm}$ ); Euclidean distances were markedly larger in the TD group compared to the HCP group for the more affected hemisphere and markedly shorter in the TD compared to the HCP for the less affected hemisphere. In the DCP children, Euclidean distances of S1 activities were markedly larger for both hemispheres compared to both hemispheres of the TD children. The Euclidean distances between activities within the contralateral S1 due to stimulation of D1, D3, and D5 for all participants are presented in Table 2 .

\section{TRACTOGRAPHY RESULTS}

Figure 4 presents the thalamocortical tracks from thalamus to post-central gyrus (upper panel) and thalamus to pre-central gyrus (lower panel) for the same individuals as in Figure 1. The number of fibers between thalamus and post-central gyrus was not noticeably different between the two hemispheres in any of the participant groups (Figure 5 - upper panel). Mean FA values for the thalamocortical fibers from thalamus to post-central gyrus were lower for the affected compared to the non-affected hemisphere in both CP groups and this difference was clearly visible in the HCP group (Figure 5 - upper panel). Mean ADC, AD, and RD values for the thalamocortical fibers from thalamus to post-central gyrus were markedly higher for the affected than the non-affected hemisphere in both CP groups, where the difference was more pronounced in the HCP group (Figure 5 - upper panel).

The mean number of fibers between thalamus and pre-central gyrus was higher in the non-affected hemisphere compared to the affected hemisphere in both CP groups and this difference was
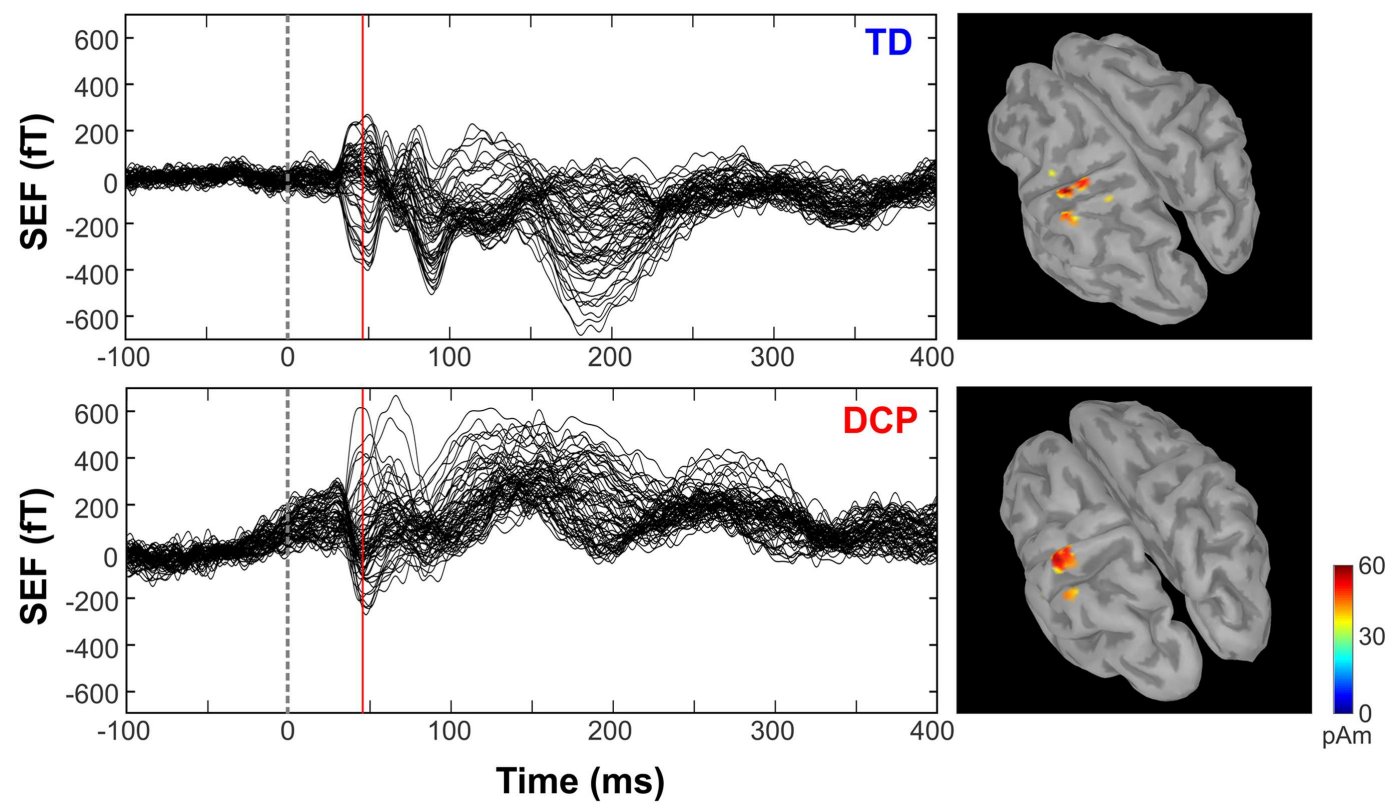

FIGURE 2 | Somatosensory-evoked fields evoked by the tactile stimulation of the right D1 (left panels) for TD1 and DCP2 (left hemisphere more affected) children respectively from $\mathbf{- 1 0 0}$ to $\mathbf{4 0 0} \mathbf{~ m s . ~ M N E s ~ ( i n ~ p i c o ~ a m p e r e ~ m e t e r ) ~ o v e r l a i d ~ o n ~ p a r t i c i p a n t ~ c o r t i c a l ~ s u r f a c e s ~ a t ~ t h e ~ p e a k ~ o f ~ M 5 0 . ~}$ 


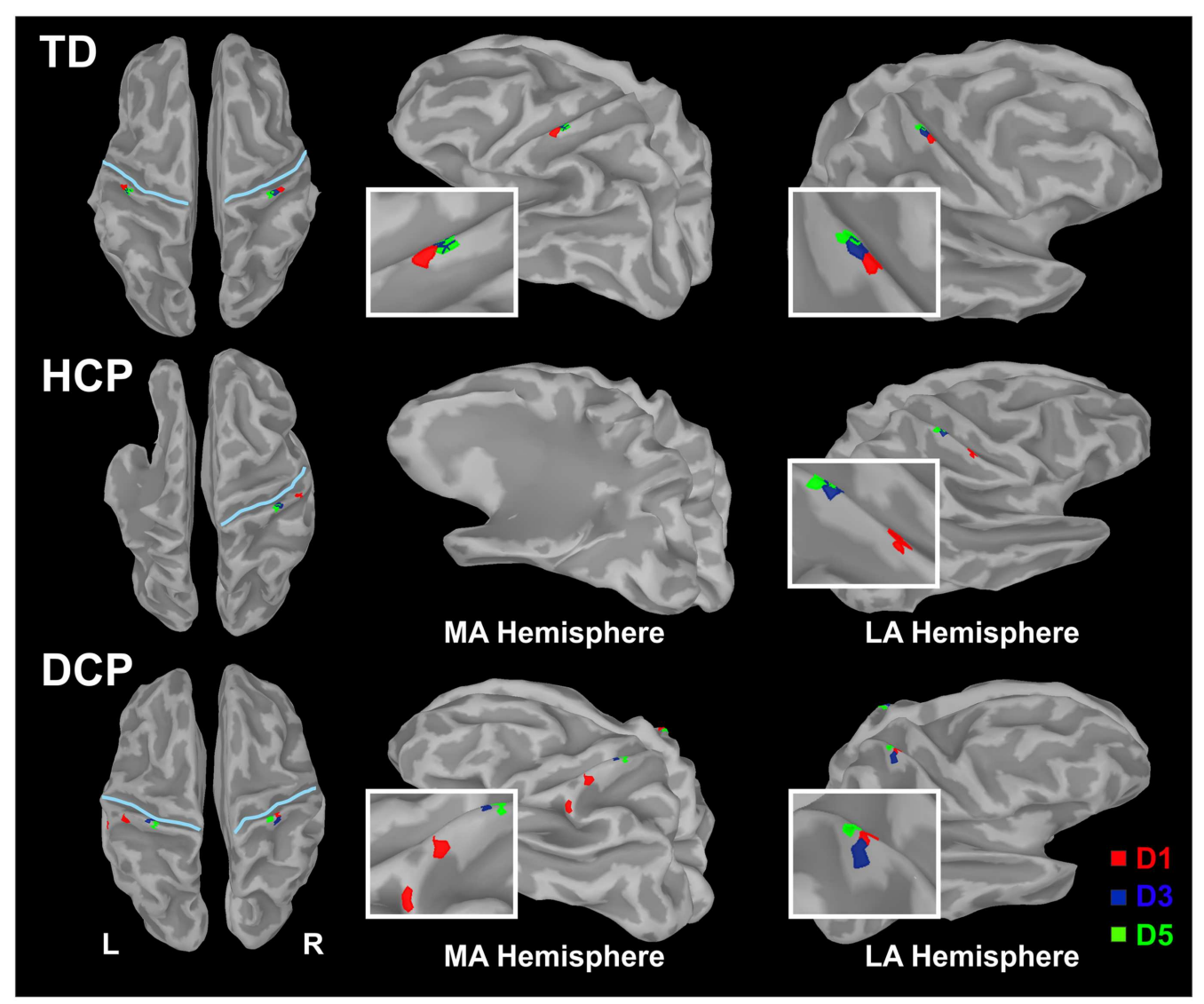

FIGURE 3 | Cortical responses in S1 at the peak of M50 for the tactile stimulation of D1, D3, and D5 of the right (left panel) and left (right panel) hands in the three representative participants (TD1, HCP1, and
DCP2). Cortical responses were defined by the maxima MNEs elicited in the contralateral hemisphere. MA hemisphere, more affected hemisphere; LA, less affected hemisphere. markedly larger in the HCP group (Figure 5 - lower panel). Mean FA values for the fibers between thalamus and pre-central gyrus were lower for the affected compared to the non-affected hemisphere in both CP groups, where the difference was most visible in the HCP group (Figure 5 - lower panel). Moreover, mean ADC, $\mathrm{AD}$, and $\mathrm{RD}$ values of the thalamocortical fibers from thalamus to pre-central gyrus were higher for the affected than the nonaffected hemisphere in both CP groups and the differences were always more pronounced in the HCP group (Figure 5 - lower panel).

Mean diffusivity values for each individual in the three participant groups mostly followed the trend that we observed in the group averages for both fiber tracts (thalamus to post-central gyrus and thalamus to pre-central gyrus). Individual results are presented in Tables 3 and 4. The most striking result belonged to the HCP child (HCP1) with the large cortical lesion on the left hemisphere. For both fiber tracts, the mean ADC, AD, and RD values for this child's affected hemisphere was markedly increased compared to the non-affected hemisphere, while the mean FA values for the affected hemisphere was notably decreased than the non-affected hemisphere. Another interesting finding was of a DCP child (DCP3), whose left leg was affected and had a normal MRI of the brain according to the radiology report. Due to the left foot paresis, this child's right hemisphere was considered to be more affected; however, the results were not straightforward. For thalamus to post-central gyrus fiber tracts, this child's affected hemisphere did indeed had a lower mean FA value than the non-affected hemisphere like the rest of the CP children, however, for thalamus to pre-central gyrus fiber tracts, mean FA value was markedly higher for the affected hemisphere than the non-affected hemisphere unlike the rest of the CP children. The mean $\mathrm{ADC}, \mathrm{AD}$, and $\mathrm{RD}$ values for this child's affected hemisphere were lower compared to the non-affected hemisphere for both fiber tracts. Nevertheless, the differences between the mean diffusivity values of the two hemispheres in DCP3 were not too large.

\section{rs-fMRI RESULTS}

The number of spatially independent component maps ranged from 76 to 98 for the nine participants. We identified four ICA maps representing two anatomically distinct RSNs associated with the sensorimotor area. RSN1 and RSN2 were unilateral SS networks in the right or the left hemisphere, and RSN3 was a bilateral SS network. Both of these networks included activation in precentral and post-central gyri, which are areas that represent the hand region in S1 and M1 in TD individuals (Liu et al., 2008). 
RSN4 was a motor network that included activation in the midline and its surrounding areas, which are shown to represent the foot and leg region in TD individuals (Luft et al., 2002; Kapreli et al., 2006; Christensen et al., 2007). Similar patterns have been identified in previous RSN studies (Biswal et al., 1995; Beckmann et al., 2005; Liu et al., 2008). Table 5 shows the RSNs identified for each participant.

Table 2 | Euclidean distances (in millimeter) for activities in S1.

\begin{tabular}{|c|c|c|c|c|c|c|}
\hline \multirow[t]{2}{*}{ Participant } & \multicolumn{2}{|c|}{ D1-D3 } & \multicolumn{2}{|c|}{ D3-D5 } & \multicolumn{2}{|c|}{ D1-D5 } \\
\hline & $\mathbf{R H}$ & LH & $\mathbf{R H}$ & LH & $\mathbf{R H}$ & LH \\
\hline TD1 & 7.79 & 7.82 & 7.79 & 14.85 & 0.00 & 7.56 \\
\hline TD2 & 9.42 & 3.72 & 10.50 & 1.00 & 14.77 & 3.72 \\
\hline TD3 & 7.13 & 8.44 & 4.35 & 2.87 & 12.11 & 10.96 \\
\hline \multirow[t]{2}{*}{ Average } & 8.11 & 6.66 & 7.54 & 6.24 & 8.96 & 7.41 \\
\hline & MA & LA & MA & LA & MA & LA \\
\hline HCP1 & $*$ & 18.41 & $*$ & 5.88 & $*$ & 24.23 \\
\hline $\mathrm{HCP} 2$ & 0.00 & 8.44 & 0.00 & 23.97 & 0.00 & 29.24 \\
\hline HCP3 & 0.00 & 12.00 & 0.00 & 1.88 & 0.00 & 10.96 \\
\hline \multirow[t]{2}{*}{ Average } & 0.00 & 12.95 & 0.00 & 10.58 & 0.00 & 21.47 \\
\hline & MA & LA & MA & LA & MA & LA \\
\hline DCP1 & 5.15 & - & - & - & - & - \\
\hline DCP2 & 20.63 & 11.31 & 28.03 & 7.54 & 10.29 & 11.55 \\
\hline DCP3 & 8.34 & 31.49 & 9.94 & 6.54 & 16.44 & 25.17 \\
\hline DCP4 & 33.82 & 10.12 & 9.71 & 11.16 & 38.36 & 18.98 \\
\hline Average & 16.98 & 17.64 & 15.89 & 8.41 & 21.69 & 18.56 \\
\hline
\end{tabular}

*, Absent activity.

-, No data.
All three SS ICA maps were present for each TD participant. Moreover, the bilateral SS network had a symmetrical pattern in all TD children. The most affected HCP participants did not have all of the three SS RSNs. HCP1, who had a large lesion in his left hemisphere did not have the left sided and the bilateral SS RSNs. HCP2 whose right hemisphere was affected, did not have separate SS RSNs for the right and left hemisphere and had an asymmetrical bilateral SS RSN where the left side activation was more widespread. HCP3 was the least affected child with HCP whose left hemisphere was affected. For him, we could find all the three SS RSNs. Nevertheless, his bilateral SS RSN was asymmetrical where the right hemisphere had a more widespread activity. We observed the fewest number of SS RSNs for the participants with DCP. DCP1 did not have either of the right and left SS RSNs, while her bilateral SS RSN was symmetrical. DCP2 did not have a right-sided SS RSN and her bilateral SS activity was larger on the left hemisphere. DCP3 did not have either of the right and left SS RSNs, and his bilateral SS RSN had symmetrical activity on both hemispheres.

The motor RSN was present in all nine participants. Withingroup average results show that the localization of activity in the TD and HCP groups was similar and more restricted to the midline. However, the activity was stronger in the HCP group than in the TD group. On the other hand, the activity was more widespread in the DCP group than the other two participant groups, also covering the primary and secondary SS regions other than the motor region, a finding, which is similar to the one found in Burton et al. (2009).

\section{DISCUSSION}

Our preliminary multimodal neuroimaging findings suggest an abnormal SS processing mechanism in children with SCP. This mechanism could be the result of a possible reorganization process. Our results evident an altered SS mechanism as: (i) an

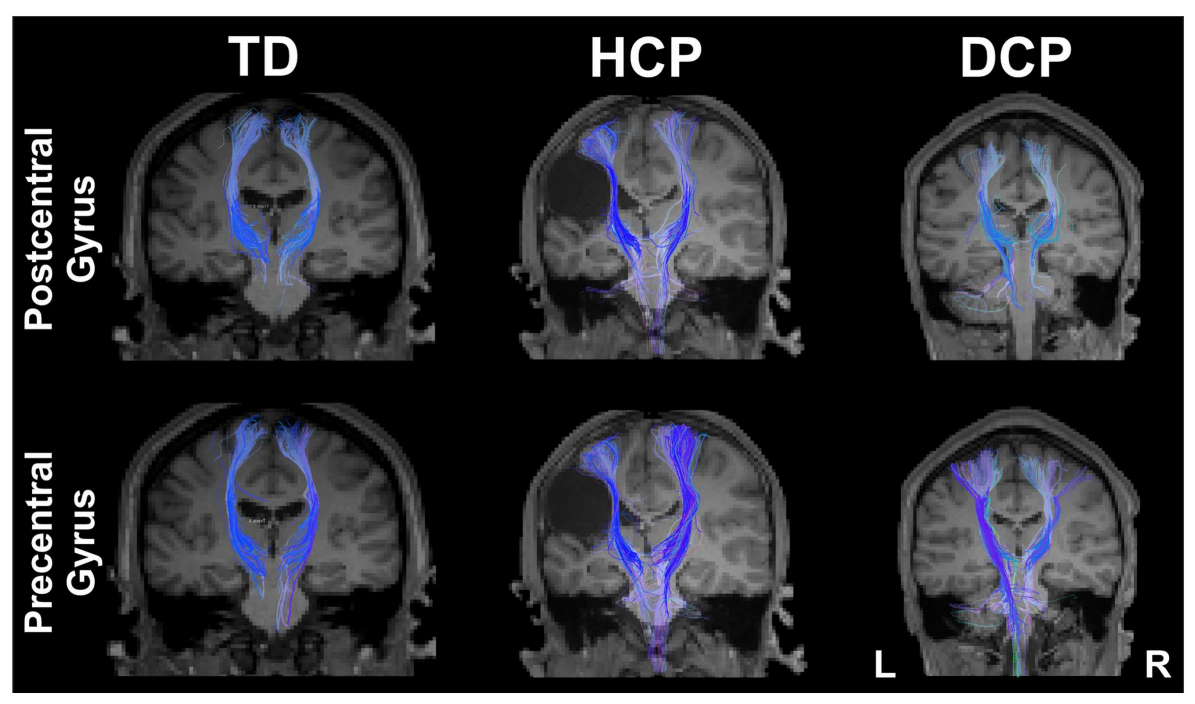

FIGURE 4 |Thalamocortical fibers projecting from thalamus to the post-central gyrus (upper panel) and from thalamus to pre-central gyrus (lower panel) for both sides of the brain for the same participants as in Figure 1 (TD2, HCP1, and DCP3 children) 


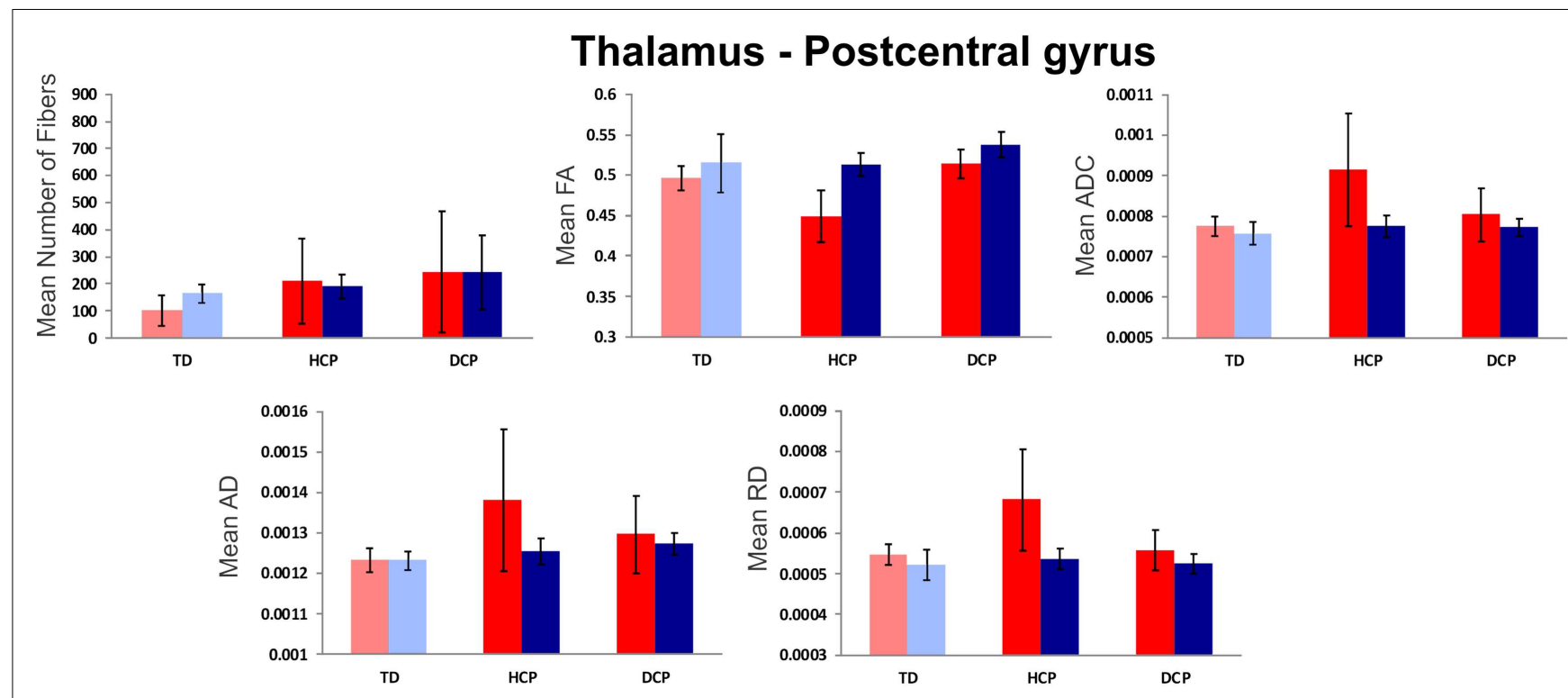

Thalamus - Precentral gyrus
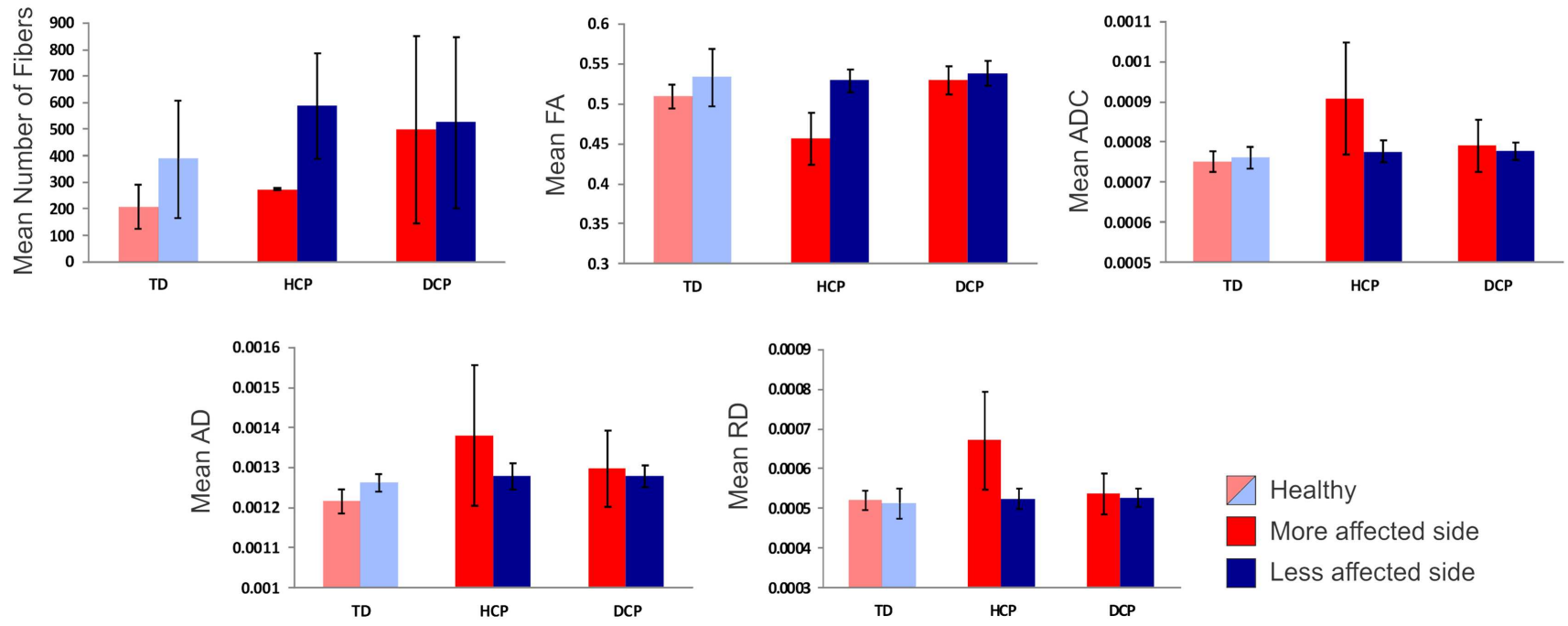

FIGURE 5 | Mean diffusivity values ( \pm SD) for the thalamocortical fibers projecting from thalamus to post-central gyrus (upper panel) and thalamus to pre-central gyrus (lower panel) for all participants. Red

indicates the affected (or more affected) hemisphere; blue indicates the intact (or less affected) hemisphere. Light red/blue indicate left and right hemisphere in TD.

altered morphology of the evoked responses elicited contralateral to the stimuli in both hemispheres of DCP and HCP children; (ii) an altered somatotopic representation of the hand areas in the contralateral S1 to the more paretic hand of two DCP children and to the non-paretic hand of one HCP child, (iii) markedly different Euclidean distances between S1 activities elicited by tactile stimulation of D1, D3, and D5 between the two hemispheres in the HCP children and between DCP and TD children for both hemispheres, and (iv) absent and/or abnormal sensorimotor RSNs for children with HCP and DCP. Functional abnormalities detected by MEG and rs-fMRI in CP children were supported by our DTI findings, which indicate structural deficits in the thalamocortical fibers projecting from thalamus to the pre-central and post-central gyri.

Functional alterations in the magnetic evoked responses observed here have also been previously reported after the tactile stimulation of fingers and after the electrical stimulation of the median nerve in HCP children with subcortical lesions (Nevalainen et al., 2012). These alterations include missing deflections, aberrant morphology, and different distances in the localized SEFs components between CP and TD patients. Such findings could be due to diminished thalamocortical projections from thalamus to S1 (Hoon et al., 2002; Lee et al., 2005) and/or subsequent aberrant functioning of cortical SS network. Our 
Table 3 | DTI results from thalamus to post-central gyrus (mean values for the more affected and less affected hemispheres) ${ }^{\mathrm{a}}$.

\begin{tabular}{|c|c|c|c|c|c|c|c|c|c|c|}
\hline Participant & \multicolumn{2}{|c|}{ NoF } & \multicolumn{2}{|c|}{ FA } & \multicolumn{2}{|c|}{$A D C$} & \multicolumn{2}{|c|}{$A D$} & \multicolumn{2}{|c|}{ RD } \\
\hline TD2 & 84 & 193 & 0.5101 & 0.5529 & 0.000757 & 0.000727 & 0.001203 & 0.001221 & 0.000535 & 0.000479 \\
\hline TD3 & 161 & 127 & 0.4969 & 0.5099 & 0.000765 & 0.000774 & 0.001231 & 0.001257 & 0.000531 & 0.000532 \\
\hline Average & 99.3 & 163 & 0.4956 & 0.5148 & 0.000775 & 0.000758 & 0.001232 & 0.001231 & 0.000547 & 0.000521 \\
\hline HCP2 & 30 & 215 & 0.4545 & 0.5233 & 0.000849 & 0.000771 & 0.001274 & 0.001260 & 0.000636 & 0.000526 \\
\hline HCP3 & 334 & 215 & 0.4789 & 0.4991 & 0.000818 & 0.000801 & 0.001285 & 0.001282 & 0.000692 & 0.000560 \\
\hline Average & 209 & 189 & 0.4492 & 0.5136 & 0.000914 & 0.000774 & 0.001380 & 0.001254 & 0.000716 & 0.000534 \\
\hline DCP1 & 491 & 387 & 0.5169 & 0.5201 & 0.000850 & 0.000797 & 0.001367 & 0.001290 & 0.000592 & 0.000551 \\
\hline
\end{tabular}

${ }^{a}$ NoF, number of fibers; FA, fractional anisotropy; $A D C$, apparent diffusion coefficient; $A D$, axial diffusivity; RD, radial diffusivity; $M A$, more affected hemisphere; $L A$, less affected hemisphere.

Table 4 | DTI results from thalamus to pre-central gyrus (mean values for the more affected and less affected hemispheres)

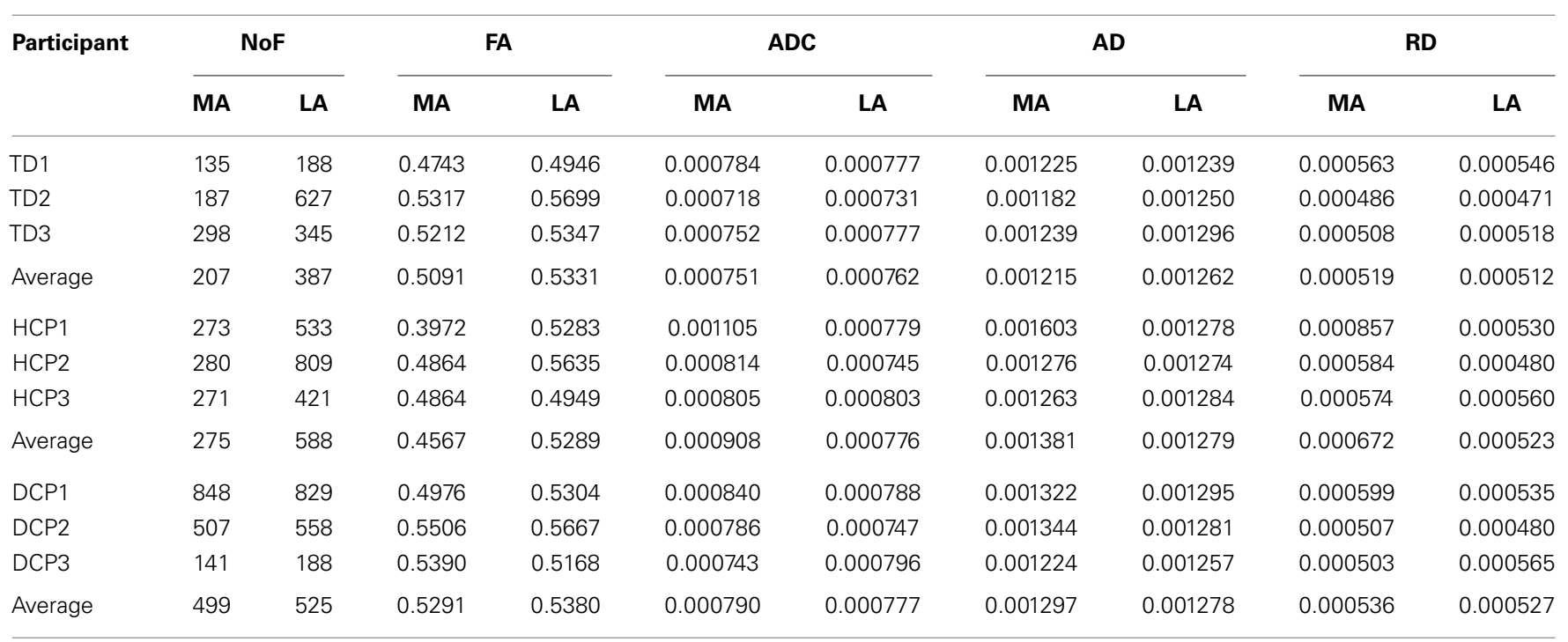

${ }^{a}$ NoF, number of fibers; $F A$, fractional anisotropy; $A D C$, apparent diffusion coefficient; $A D$, axial diffusivity; $R D$, radial diffusivity; $M A$, more affected hemisphere; $L A$, less affected hemisphere.

Table 5 | Somatosensory (SS) and motor RSNs.

\begin{tabular}{|c|c|c|c|c|c|c|}
\hline Participant & Affected side & Components & SS right & SS left & SS bilateral & Motor \\
\hline TD2 & N/A & 76 & + & + & Symmetrical & + \\
\hline HCP1 & Right & 81 & + & - & - & + \\
\hline $\mathrm{HCP} 2$ & Left & 89 & - & - & Asymmetrical (left larger) & + \\
\hline HCP3 & Right & 91 & + & + & Asymmetrical (right larger) & + \\
\hline DCP2 & Both (right more) & 88 & - & + & Asymmetrical (left larger) & + \\
\hline DCP3 & Both (left more) & 79 & - & - & Symmetrical & + \\
\hline
\end{tabular}


Euclidean distance results are in line with Nevalainen et al. (2012) that reported significant different distances between the representation areas of D1, D3, and D5 in the more affected hemisphere of HCP children compared to TD. Our most striking MEG finding was the altered somatotopy in the hemisphere contralateral to the most paretic hand in two DCP children and to the non-paretic hand in one HCP child. To our best knowledge, it is the first time that an altered somatotopic order is reported in CP.

In contrast with previous EEG and MEG studies (Teflioudi et al., 2011; Guo et al., 2012; Pihko et al., 2014), but in line with others (Nevalainen et al., 2012), we did not observe differences in latencies of the first prominent cortical response at M50 between the $\mathrm{CP}$ and $\mathrm{TD}$ children. Alterations in later deflections that were observed bilaterally in the DCP children likely reflect aberrant processing in the local cortical network after the arrival of the thalamocortical input to the cortex. Guo et al. (2012) reported delayed latencies for the first SEFs response in CP compared to TD children. Our group also observed similar findings in an adult $\mathrm{CP}$ patient with a large unilateral, prenatally acquired, periventricular brain lesion (Papadelis et al., 2012). On the other hand, other studies have reported the first cortical response at normal latencies after tactile stimulation of the paretic thumb of $\mathrm{CP}$ children with PVL, localized in its original topography in the Rolandic region of the affected hemisphere (Gerloff et al., 2006; Staudt et al., 2006; Wilke and Staudt, 2009). The contradictory findings between all these studies might be explained by the possible different timing of the insults in these patients. The maturational stage of the brain at the time of the insult determines the type of structural pathology and eventually the possible reorganization mechanism (Staudt, 2007).

One of our HCP children (HCP1) had a large lesion on his left hemisphere due to prenatal left middle cerebral artery infarct. For this child, the M50 component was absent from the SEFs in the contralateral hemisphere when the digits of the paretic hand were stimulated. Missing early cortical responses have been previously reported by Pihko et al. (2014) after the stimulation of the median nerve in CP children. There are two possible mechanisms that can explain our finding for the HCP1: (i) the primary SS representation of the paretic hand has been transferred to the contralesional hemisphere, ipsilateral to the stimulation due to damaged projections of the afferent thalamocortical fibers to S1, or (ii) the afferent thalamocortical SS projections had apparently "bypassed" the lesion and reached their original cortical destination area in the post-central gyrus, but the bypass caused a significant delay in the first cortical response that appeared later in time (see Figure 1 - left middle panel). Unfortunately, the partial coverage of our system's sensor array does not allow us to conclude which mechanism was present but only to speculate. A previous study by Staudt et al. (2006) in hemiparetic patients has supported the later explanation as the most possible one. It was found that despite the large periventricular lesions of their patients, the S1 representation of the paretic hand was located in the Rolandic cortex of their affected hemisphere. They assumed that the outgrowing thalamocortical projections might have developed as a consequence of compensation after the insult. In our HCP1 patient, these axons may not have found their way to the preserved tissue around the periventricular defect and may project to the ipsilateral hemisphere. Although this is a plausible explanation, the exact timing of the insult remains unclear in all these studies. A later cortical response peaking at $\sim 80 \mathrm{~ms}$ was observed in this child that was localized in the pre-central gyrus. This response was prolonged looking similar to components that occur at later latencies ( $\sim 80-100 \mathrm{~ms}$ after stimulus onset) in the normal SS processing and probably reflects secondary processes in the SS cortex. The localization of this component in the pre-central gyrus might be explained by the missing secondary SS cortex in the lesioned hemisphere. Such a later component was also present in the TD and DCP children at the same latency (see Figure 1).

We further used two neuroimaging methods, namely DTI and rs-fMRI, that can help us disentangle this issue. Several previous studies have used DTI to investigate microstructural abnormalities responsible for motor weakness and disability in children with $\mathrm{CP}$ because conventional MRI is unable to detect subtle structural abnormalities (Lee et al., 2003). However, to our knowledge, no study yet reported the integrity of motor and sensory white matter pathway differences among TD, HCP, and DCP children. In our study, we found decreased FA values for the thalamocortical pathways in the affected (compared to the unaffected) hemisphere in children with $\mathrm{CP}$, which may reflect a loss or disorganization of the structural barriers to molecular diffusion of water in these patients (Trivedi et al., 2010; Rai et al., 2013). Increased ADC values could be suggestive of increased extracellular water content due to gliosis and microscopic cystic changes emerging at the affected brain regions in CP children (Trivedi et al., 2010; Rai et al., 2013). In our findings, the increase in ADC values was accompanied by an increase both in $\mathrm{AD}$ and, primarily, $\mathrm{RD}$. Studies have shown that while an increase in RD might be a sign of disorganized, demyelinated, dysmyelinated, and/or poorly myelinated axons (Song et al., 2002, 2005; Nair et al., 2005), an increase in AD is associated with axonal injury or damage, which causes a decrease in axonal density or caliber, finally resulting in an increase in the extra-axonal space allowing water molecules to move faster (Song et al., 2005; Sun et al., 2008; Kumar et al., 2010; Della Nave et al., 2011).

We found that children with HCP showed the most noticeable FA and ADC value differences between their affected and non-affected hemispheres for both of the pathways that we studied. The mean number of tracts was considerably higher for the non-affected hemisphere of HCP children compared to their affected hemisphere and to the healthy hemispheres of TD children. Although this change does not necessarily directly translate into an increase in the actual number of axons (Koerte et al., 2011), it may indicate a possible reorganization of the thalamus to the pre-central gyrus. Our finding is in parallel with previous studies, which showed that quadriplegic $\mathrm{CP}$ children were more affected (decreased mean FA and increased mean ADC in corticospinal tract) than DCP (Chang et al., 2012), and CP children with low gross motor function were more affected (lower number of fibers in corticospinal tract) than ones with high gross motor function (Rha et al., 2012).

Our DTI results indicate anatomical deficits in both the quality and quantity of thalamocortical fibers projecting from thalamus to the pre-central and post-central gyri. The diminished quality of the ascending thalamocortical fibers projecting from thalamus to the post-central gyrus may explain the observed abnormalities in 
the SEFs. Our DTI findings also support the notion that anatomical deficits in the sensory pathways, in addition to the motor pathways, could be responsible for the pathophysiology of motor disability and weakness in children with CP. By extending the findings of previous studies, we show a clear difference in the sensory and motor pathways of children with HCP compared to DCP and TD children, as well as an indication of possible reorganization of the tracks from thalamus to pre-central gyrus in HCP children.

In order to test the functionality of the cortical SS network in $\mathrm{CP}$ children, we have also examined the sensorimotor RSNs in the three groups of children using an ICA method. For TD children, we managed to identify all the known SS and motor RSNs. HCP and DCP children showed absent and/or abnormal sensorimotor RSNs consistent with the severity and location of their lesions. Participants with HCP had absent or weak SS networks on their lesioned hemispheres while the intact hemisphere seemed to have stronger and more widespread activation (see Figure 6). Participants with DCP showed widespread activity for the motor RSN, extending to the SS cortices compared to the other participant groups, which is a similar finding as in Burton et al. (2009). The more widespread and/or stronger activity in participants with CP could be explained by disruptions to the somatotopic organizations in the SS cortices and the development of new and the strengthening of already existing intracortical connections (Marin-Padilla, 1997; Burton et al., 2009). The findings are in parallel with our DTI findings where the number of fibers for DCP children was low for the thalamus and post-central pathway but high for thalamus to pre-central pathway for both hemispheres.

\section{METHODOLOGICAL LIMITATIONS}

Magnetoencephalography findings were used to determine the S1 functionally for the DTI analysis. In all CP and TD children, S1 activity was localized within the post-central gyrus. Ideally, the MEG-defined ROIs should be used for the determination of ROIs in the DTI analysis. However, fiber tracking has limitations in determining the thalamocortical SS radiations and the motor tracts for the hand areas due to its well-known weakness to handle crossing fibers at the centrum semiovale level (Nowinski et al., 2012). Here, we were not able to reconstruct fibers passing from the MEG-defined ROIs to the thalamus. Therefore, for the DTI analysis, we considered the volume of the entire post-central gyrus as the ROI. Since our experimental setup did not involve any spontaneous movements from the participants, we were unable to determine the M1 functionally. Although MNE indicated cortical activity within the pre-central gyrus, this activity was not considered to be significant functionally. There is evidence that MNE solutions of even focal sources can extend across sulcal walls separated by only a few millimeters (Liu et al., 2002; Lin et al., 2006b; Hauk et al., 2011). The observed activity within the precentral gyrus was probably due to the expansion of MNE solutions from the actual primary generator located within the post-central gyrus to more anterior locations. Since we were unable to functionally define the M1 by using our MEG data, we followed the most common practice to define it anatomically by choosing the volume of the entire pre-central gyrus as the ROI (Trivedi et al., 2010; Chang et al., 2012; Rha et al., 2012).

\section{CONCLUSION}

Even though our study is limited by the relatively small number of participants and the lack of statistical results, it is the first study that reports findings from multiple neuroimaging modalities in the same CP patients. Our functional and anatomical findings provide preliminary evidence of impaired SS processing in $\mathrm{CP}$ patients that are due to (i) diminished thalamocortical projections from thalamus to S1 (Hoon et al., 2002; Lee et al., 2005), and (ii) subsequent aberrant functioning of the cortical SS network.

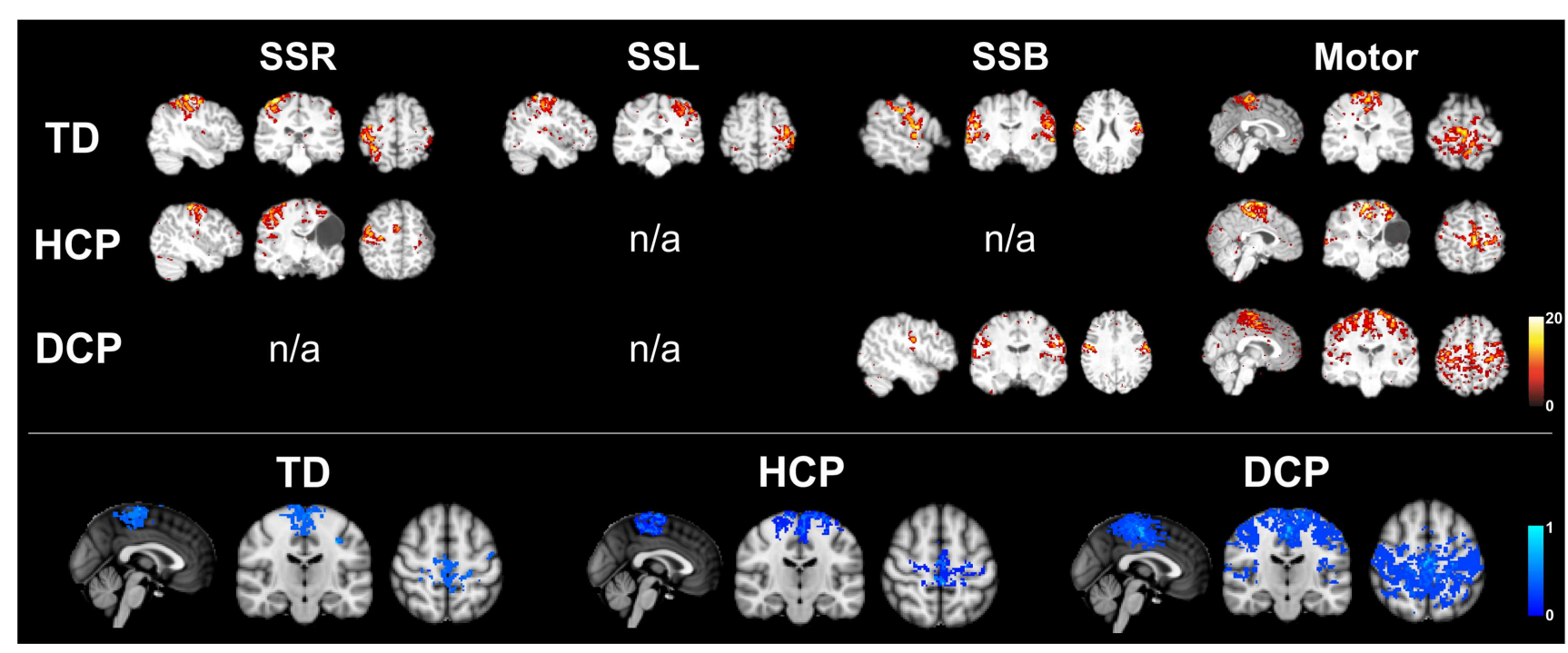

FIGURE 6 | Upper panel: RSNs for the same participants as in Figure 1 (TD2, HCP1, and DCP3 children). SSR, right somatosensory RSN; SSL, left somatosensory RSN; SSB, bilateral somatosensory RSN; motor, motor RSN (z-scores). Lower panel: mean motor RSN (lower panel) for the three groups of participants. 
It should be noted though that our findings are limited due to the partial head coverage of our BabySQUID MEG system that does not allow simultaneous recordings of both hemispheres. Also, our small sample size prevents us from generalizing our conclusions to the general CP population. We conclude that motor and sensory pathways could both be important in the clinical outcome of children with $\mathrm{CP}$ and that the diminished connectivity in these pathways in the affected hemisphere, especially of children with HCP, could be indicative of a pathophysiological mechanism responsible for motor dysfunction (Yoshida et al., 2010; Lee et al., 2011). These results have important implications for the diagnosis and the rehabilitation of patients with CP. Such interventions should be applied early in life when the brain demonstrates the ability to plasticity, allowing it to readily reorganize in the face of injury (Johnston, 2009). Therefore, new approaches using innovative technologies that will identify early functional and structural deficits in the brain of children with PVL are needed.

\section{ACKNOWLEDGMENTS}

The authors would like to thank Chiran Doshi, MSc for his technical support with the data analysis and collection, Tapsya Nayak, MSc for her help with the data collection, Borjan Gagoski, Ph.D. for his help with the MRI sequences, Mathieu Dehaes, Ph.D., and Danielle Sliva, MA, for their help with rs-fMRI analysis.

\section{REFERENCES}

Bax, M., Goldstein, M., Rosenbaum, P., Leviton, A., Paneth, N., Dan, B., et al. (2005). Proposed definition and classification of cerebral palsy, April 2005. Dev. Med. Child Neurol. 47, 571-576. doi:10.1017/S001216220500112X

Beckmann, C. F., DeLuca, M., Devlin, J. T., and Smith, S. M. (2005). Investigations into resting-state connectivity using independent component analysis. Philos. Trans. R. Soc. Lond. B Biol. Sci. 360, 1001-1013. doi:10.1098/rstb.2005.1634

Beckmann, C. F., and Smith, S. M. (2004). Probabilistic independent component analysis for functional magnetic resonance imaging. IEEE Trans. Med. Imaging 23, 137-152. doi:10.1109/TMI.2003.822821

Biswal, B., Yetkin, F. Z., Haughton, V. M., and Hyde, J. S. (1995). Functional connectivity in the motor cortex of resting human brain using echo-planar MRI. Magn. Reson. Med. 34, 537-541. doi:10.1002/mrm.1910340409

Burton, H., Dixit, S., Litkowski, P., and Wingert, J. R. (2009). Functional connectivity for somatosensory and motor cortex in spastic diplegia. Somatosens. Mot. Res. 26, 90-104. doi:10.3109/08990220903335742

Chang, M. C., Jang, S. H., Yoe, S. S., Lee, E., Kim, S., Lee, D. G., et al. (2012). Diffusion tensor imaging demonstrated radiologic differences between diplegic and quadriplegic cerebral palsy. Neurosci. Lett. 512, 53-58. doi:10.1016/j.neulet.2012. 01.065

Christensen, M. S., Lundbye-Jensen, J., Petersen, N., Geertsen, S. S., Paulson, O. B., and Nielsen, J. B. (2007). Watching your foot move - an fMRI study of visuomotor interactions during foot movement. Cereb. Cortex 17, 1906-1917. doi:10.1093/cercor/bhl101

Cooper, J., Majnemer, A., Rosenblatt, B., and Birnbaum, R. (1995). The determination of sensory deficits in children with hemiplegic cerebral palsy. J. Child Neurol. 10, 300-309. doi:10.1177/088307389501000412

Coq, J. O., Strata, F., Russier, M., Safadi, F. F., Merzenich, M. M., Byl, N. N., et al. (2008). Impact of neonatal asphyxia and hind limb immobilization on musculoskeletal tissues and S1 map organization: implications for cerebral palsy. Exp. Neurol. 210, 95-108. doi:10.1016/j.expneurol.2007.10.006

Daducci, A., Gerhard, S., Griffa, A., Lemkaddem, A., Cammoun, L., Gigandet, X., et al. (2012). The connectome mapper: an open-source processing pipeline to map connectomes with MRI. PLOS ONE 7:e48121. doi:10.1371/journal.pone. 0048121

Dale, A. M., Fischl, B., and Sereno, M. I. (1999). Cortical surface-based analysis. I. Segmentation and surface reconstruction. Neuroimage 9, 179-194. doi:10.1006/ nimg.1998.0395
Dehaes, M., Raschle, N. M., Sliva, D. D., Zuk, J., Drottar, M., Chang, M., et al. (2013). Potential of Resting State Connectivity and Passive fMRI to Detect Precursors of Learning Disabilities in Infants: Preliminary Results with Infants at Familial Risk for Developmental Dyslexia. Salt Lake City: The International Society for Magnetic Resonance in Medicine.

Della Nave, R., Ginestroni, A., Diciotti, S., Salvatore, E., Soricelli, A., and Mascalchi, M. (2011). Axial diffusivity is increased in the degenerating superior cerebellar peduncles of Friedreich's ataxia. Neuroradiology 53, 367-372. doi:10.1007/s00234-010-0807-1

Dijkerman, H. C., and de Haan, E. H. (2007). Somatosensory processes subserving perception and action. Behav. Brain Sci. 30, 189-201. doi:10.1017/ S0140525X07001392 discussion 201-139,

Einspieler, C., and Prechtl, H. F. (2005). Prechtl's assessment of general movements: a diagnostic tool for the functional assessment of the young nervous system. Ment. Retard. Dev. Disabil. Res. Rev. 11, 61-67. doi:10.1002/ mrdd.20051

Fischl, B., Liu, A., and Dale, A. M. (2001). Automated manifold surgery: constructing geometrically accurate and topologically correct models of the human cerebral cortex. IEEE Trans. Med. Imaging 20, 70-80. doi:10.1109/42.906426

Fischl, B., Sereno, M. I., and Dale, A. M. (1999). Cortical surface-based analysis. II: inflation, flattening, and a surface-based coordinate system. Neuroimage 9, 195-207. doi:10.1006/nimg.1998.0396

Fowler, E. G., and Goldberg, E. J. (2009). The effect of lower extremity selective voluntary motor control on interjoint coordination during gait in children with spastic diplegic cerebral palsy. Gait Posture 29, 102-107. doi:10.1016/j.gaitpost. 2008.07.007

Gerloff, C., Bushara, K., Sailer, A., Wassermann, E. M., Chen, R., Matsuoka, T., et al. (2006). Multimodal imaging of brain reorganization in motor areas of the contralesional hemisphere of well recovered patients after capsular stroke. Brain 129, 791-808. doi:10.1093/brain/awh713

Gordon, A. M., Bleyenheuft, Y., and Steenbergen, B. (2013). Pathophysiology of impaired hand function in children with unilateral cerebral palsy. Dev. Med. Child Neurol. 55(Suppl. 4), 32-37. doi:10.1111/dmcn.12304

Guo, X., Xiang, J., Mun-Bryce, S., Bryce, M., Huang, S., Huo, X., et al. (2012). Aberrant high-gamma oscillations in the somatosensory cortex of children with cerebral palsy: a meg study. Brain Dev. 34, 576-583. doi:10.1016/j.braindev.2011.09. 012

Hadders-Algra, M. (2004). General movements: a window for early identification of children at high risk for developmental disorders. J. Pediatr. 145, S12-S18. doi:10.1016/j.jpeds.2004.05.017

Hämäläinen, M., Hari, R., Ilmoniemi, R., Knuutila, J., and Lounasmaa, O. (1993). Magnetoencephalography - theory, instrumentation, and applications to noninvasive studies of the working human brain. Rev. Mod. Phys. 65, 1-93.

Hamalainen, M. S., and Ilmoniemi, R. J. (1994). Interpreting magnetic fields of the brain: minimum norm estimates. Med. Biol. Eng. Comput. 32, 35-42. doi:10.1007/BF02512476

Hauk, O., Wakeman, D. G., and Henson, R. (2011). Comparison of noise-normalized minimum norm estimates for MEG analysis using multiple resolution metrics. Neuroimage 54, 1966-1974. doi:10.1016/j.neuroimage.2010.09.053

Himmelmann, K., Beckung, E., Hagberg, G., and Uvebrant, P. (2006). Gross and fine motor function and accompanying impairments in cerebral palsy. Dev. Med. Child Neurol. 48, 417-423. doi:10.1017/S0012162206000922

Hoon, A. H. Jr., Lawrie, W. T. Jr., Melhem, E. R., Reinhardt, E. M., Van Zijl, P. C., Solaiyappan, M., et al. (2002). Diffusion tensor imaging of periventricular leukomalacia shows affected sensory cortex white matter pathways. Neurology 59, 752-756. doi:10.1212/WNL.59.5.752

Hoon, A. H. Jr., Stashinko, E. E., Nagae, L. M., Lin, D. D., Keller, J., Bastian, A., et al. (2009). Sensory and motor deficits in children with cerebral palsy born preterm correlate with diffusion tensor imaging abnormalities in thalamocortical pathways. Dev. Med. Child Neurol. 51, 697-704. doi:10.1111/j.1469-8749. 2009.03306.x

Hsiao, F. J., Cheng, C. H., Chen, W. T., and Lin, Y. Y. (2013). Neural correlates of somatosensory paired-pulse suppression: a MEG study using distributed source modeling and dynamic spectral power analysis. Neuroimage 72, 133-142. doi:10.1016/j.neuroimage.2013.01.041

Huang, M. X., Mosher, J. C., and Leahy, R. M. (1999). A sensor-weighted overlappingsphere head model and exhaustive head model comparison for MEG. Phys. Med. Biol. 44, 423-440. doi:10.1088/0031-9155/44/2/010 
Jenkinson, M., Bannister, P., Brady, M., and Smith, S. (2002). Improved optimization for the robust and accurate linear registration and motion correction of brain images. Neuroimage 17, 825-841. doi:10.1006/nimg.2002.1132

Johnston, M. V. (2009). Plasticity in the developing brain: implications for rehabilitation. Dev. Disabil. Res. Rev. 15, 94-101. doi:10.1002/ddrr.64

Kapreli, E., Athanasopoulos, S., Papathanasiou, M., Van Hecke, P., Strimpakos, N., Gouliamos, A., et al. (2006). Lateralization of brain activity during lower limb joints movement. An fMRI study. Neuroimage 32, 1709-1721. doi:10.1016/j. neuroimage.2006.05.043

Koerte, I., Pelavin, P., Kirmess, B., Fuchs, T., Berweck, S., Laubender, R. P., et al. (2011). Anisotropy of transcallosal motor fibres indicates functional impairment in children with periventricular leukomalacia. Dev. Med. Child Neurol. 53, 179-186. doi:10.1111/j.1469-8749.2010.03840.x

Krageloh-Mann, I., and Cans, C. (2009). Cerebral palsy update. Brain Dev. 31, 537-544. doi:10.1016/j.braindev.2009.03.009

Krumlinde-Sundholm, L., and Eliasson, A. C. (2002). Comparing tests of tactile sensibility: aspects relevant to testing children with spastic hemiplegia. Dev. Med. Child Neurol. 44, 604-612. doi:10.1111/j.1469-8749.2002.tb00845.x

Kumar, A., Sundaram, S. K., Sivaswamy, L., Behen, M. E., Makki, M. I., Ager, J., et al. (2010). Alterations in frontal lobe tracts and corpus callosum in young children with autism spectrum disorder. Cereb. Cortex 20, 2103-2113. doi:10.1093/cercor/bhp278

Kurz, M. J., and Wilson, T. W. (2011). Neuromagnetic activity in the somatosensory cortices of children with cerebral palsy. Neurosci. Lett. 490, 1-5. doi:10.1016/j. neulet.2010.11.053

Lee, J. D., Park, H. J., Park, E. S., Oh, M. K., Park, B., Rha, D. W., et al. (2011). Motor pathway injury in patients with periventricular leukomalacia and spastic diplegia. Brain 134, 1199-1210. doi:10.1093/brain/awr021

Lee, S. K., Kim, D. I., Kim, J., Kim, D. J., Kim, H. D., Kim, D. S., et al. (2005). Diffusion-tensor MR imaging and fiber tractography: a new method of describing aberrant fiber connections in developmental CNS anomalies. Radiographics 25, 53-65. doi:10.1148/rg.251045085 discussion 66-58,

Lee, Z. I., Byun, W. M., Jang, S. H., Ahn, S. H., Moon, H. K., and Chang, Y. (2003). Diffusion tensor magnetic resonance imaging of microstructural abnormalities in children with brain injury. Am. J. Phys. Med. Rehabil. 82, 556-559. doi:10.1097/01.PHM.0000073830.15643.6A

Prechtl, H. F., Einspieler, C., Cioni, G., Bos, A. F., Ferrari, F., and Sontheimer, D. (1997). An early marker for neurological deficits after perinatal brain lesions. Lancet 349, 1361-1363. doi:10.1016/S0140-6736(96)10182-3

Lin, F. H., Belliveau, J. W., Dale, A. M., and Hamalainen, M. S. (2006a). Distributed current estimates using cortical orientation constraints. Hum. Brain Mapp. 27, 1-13. doi:10.1002/hbm.20155

Lin, F. H., Witzel, T., Ahlfors, S. P., Stufflebeam, S. M., Belliveau, J. W., and Hämäläinen, M. S. (2006b). Assessing and improving the spatial accuracy in MEG source localization by depth-weighted minimum-norm estimates. Neuroimage 31, 160-171. doi:10.1016/j.neuroimage.2005.11.054

Liu, A. K., Dale, A. M., and Belliveau, J. W. (2002). Monte Carlo simulation studies of EEG and MEG localization accuracy. Hum. Brain Mapp. 16, 47-62. doi: $10.1002 / \mathrm{hbm} .10024$

Liu, W. C., Flax, J. F., Guise, K. G., Sukul, V., and Benasich, A. A. (2008). Functional connectivity of the sensorimotor area in naturally sleeping infants. Brain Res. 1223, 42-49. doi:10.1016/j.brainres.2008.05.054

Luft, A. R., Smith, G. V., Forrester, L., Whitall, J., Macko, R. F., Hauser, T. K., et al. (2002). Comparing brain activation associated with isolated upper and lower limb movement across corresponding joints. Hum. Brain Mapp. 17, 131-140. doi:10.1002/hbm.10058

Marin-Padilla, M. (1997). Developmental neuropathology and impact of perinatal brain damage. II. White matter lesion of the neocortex. J. Neuropathol. Exp. Neurol. 56, 219-235. doi:10.1097/00005072-199703000-00001

Nagae, L. M., Hoon, A. H. Jr., Stashinko, E., Lin, D., Zhang, W., Levey, E., et al. (2007). Diffusion tensor imaging in children with periventricular leukomalacia: variability of injuries to white matter tracts. AJNR Am. J. Neuroradiol. 28, 1213-1222. doi:10.3174/ajnr.A0534

Nair, G., Tanahashi, Y., Low, H. P., Billings-Gagliardi, S., Schwartz, W. J., and Duong, T. Q. (2005). Myelination and long diffusion times alter diffusion-tensorimaging contrast in myelin-deficient shiverer mice. Neuroimage 28, 165-174. doi:10.1016/j.neuroimage.2005.05.049

Nevalainen, P., Pihko, E., Maenpaa, H., Valanne, L., Nummenmaa, L., and Lauronen, L. (2012). Bilateral alterations in somatosensory cortical processing in hemiplegic cerebral palsy. Dev. Med. Child Neurol. 54, 361-367. doi:10.1111/j. 1469-8749.2011.04165.x

Nowinski, W. L., Chua, B. C., Yang, G. L., and Qian, G. Y. (2012). Three-dimensional interactive and stereotactic human brain atlas of white matter tracts. Neuroinformatics 10, 33-55. doi:10.1007/s12021-011-9118-x

Okada, Y., Pratt, K., Atwood, C., Mascarena, A., Reineman, R., Nurminen, J., et al. (2006). BabySQUID: a mobile, high-resolution multichannel MEG system for neonatal brain assessment. Rev. Sci. Instrum. 77, 24301-24309. doi:10.1063/1. 2168672

Ostensjo, S., Carlberg, E. B., and Vollestad, N. K. (2004). Motor impairments in young children with cerebral palsy: relationship to gross motor function and everyday activities. Dev. Med. Child Neurol. 46, 580-589. doi:10.1111/j.14698749.2004.tb01021.x

Palisano, R., Rosenbaum, P., Walter, S., Russell, D., Wood, E., and Galuppi, B. (1997). Development and reliability of a system to classify gross motor function in children with cerebral palsy. Dev. Med. Child Neurol. 39, 214-223. doi:10.1111/j.1469-8749.1997.tb07414.x

Papadelis, C., Chellamani, H., Ahtam, B., Doshi, C., Grant, E., and Okada, Y. (2013). Current and emerging potential for magnetoencephalography in pediatric epilepsy. J. Pediatr. Epilepsy 2, 73-85. doi:10.3233/PEP13040

Papadelis, C., Leonardelli, E., Staudt, M., and Braun, C. (2012). Can magnetoencephalography track the afferent information flow along white matter thalamocortical fibers? Neuroimage 60, 1092-1105. doi:10.1016/j.neuroimage.2012.01. 054

Papadelis, C., Poghosyan, V., Fenwick, P. B., and Ioannides, A. A. (2009). MEG's ability to localise accurately weak transient neural sources. Clin. Neurophysiol. 120, 1958-1970. doi:10.1016/j.clinph.2009.08.018

Pihko, E., Nevalainen, P., Vaalto, S., Laaksonen, K., Mäenpää, H., Valanne, L., et al. (2014). Reactivity of sensorimotor oscillations is altered in children with hemiplegic cerebral palsy: a magnetoencephalographic study. Hum. Brain Mapp. 35, 4105-4117. doi:10.1002/hbm. 22462

Prechtl, H. F. (1997). State of the art of a new functional assessment of the young nervous system. An early predictor of cerebral palsy. Early Hum. Dev. 50, 1-11. doi:10.1016/S0378-3782(97)00088-1

Rai, Y., Chaturvedi, S., Paliwal, V. K., Goyal, P., Chourasia, A., Singh Rathore, R. K., et al. (2013). DTI correlates of cognition in term children with spastic diplegic cerebral palsy. Eur. J. Paediatr. Neurol. 17, 294-301. doi:10.1016/j.ejpn.2012.11. 005

Rha, D. W., Chang, W. H., Kim, J., Sim, E. G., and Park, E. S. (2012). Comparing quantitative tractography metrics of motor and sensory pathways in children with periventricular leukomalacia and different levels of gross motor function. Neuroradiology 54, 615-621. doi:10.1007/s00234-011-0996-2

Riquelme, I., Cifre, I., and Montoya, P. (2011). Age-related changes of pain experience in cerebral palsy and healthy individuals. Pain Med. 15, 535-545. doi:10.1111/j.1526-4637.2011.01094.x

Riquelme, I., and Montoya, P. (2010). Developmental changes in somatosensory processing in cerebral palsy and healthy individuals. Clin. Neurophysiol. 121, 1314-1320. doi:10.1016/j.clinph.2010.03.010

Riquelme, I., Padrón, I., Cifre, I., González-Roldán, A. M., and Montoya, P. (2014). Differences in somatosensory processing due to dominant hemispheric motor impairment in cerebral palsy. BMC Neurosci. 15:10. doi:10.1186/14712202-15-10

Sanger, T. D., and Kukke, S. N. (2007). Abnormalities of tactile sensory function in children with dystonic and diplegic cerebral palsy. J. Child Neurol. 22, 289-293. doi:10.1177/0883073807300530

Smith, S. M. (2002). Fast robust automated brain extraction. Hum. Brain Mapp. 17, 143-155. doi:10.1002/hbm.10062

Son, S. M., Ahn, Y. H., Sakong, J., Moon, H. K., Ahn, S. H., Lee, H., et al. (2007). Diffusion tensor imaging demonstrates focal lesions of the corticospinal tract in hemiparetic patients with cerebral palsy. Neurosci. Lett. 420, 34-38. doi:10.1016/j.neulet.2007.04.054

Song, S. K., Sun, S. W., Ramsbottom, M. J., Chang, C., Russell, J., and Cross, A. H. (2002). Dysmyelination revealed through MRI as increased radial (but unchanged axial) diffusion of water. Neuroimage 17, 1429-1436. doi:10.1006/ nimg.2002.1267

Song, S. K., Yoshino, J., Le, T. Q., Lin, S. J., Sun, S. W., Cross, A. H., et al. (2005). Demyelination increases radial diffusivity in corpus callosum of mouse brain. Neuroimage 26, 132-140. doi:10.1016/j.neuroimage.2005.01.028 
Staudt, M. (2007). (Re-)organization of the developing human brain following periventricular white matter lesions. Neurosci. Biobehav. Rev. 31, 1150-1156. doi:10.1016/j.neubiorev.2007.05.005

Staudt, M., Braun, C., Gerloff, C., Erb, M., Grodd, W., and Krageloh-Mann, I. (2006). Developing somatosensory projections bypass periventricular brain lesions. Neurology 67, 522-525. doi:10.1212/01.wnl.0000227937.49151.fd

Sun, S. W., Liang, H. F., Cross, A. H., and Song, S. K. (2008). Evolving Wallerian degeneration after transient retinal ischemia in mice characterized by diffusion tensor imaging. Neuroimage 40, 1-10. doi:10.1016/j.neuroimage.2007. 11.049

Tadel, F., Baillet, S., Mosher, J. C., Pantazis, D., and Leahy, R. M. (2011). Brainstorm: a user-friendly application for MEG/EEG analysis. Comput. Intell. Neurosci. 2011, 879716. doi:10.1155/2011/879716

Teflioudi, E. P., Zafeiriou, D. I., Vargiami, E., Kontopoulos, E., and Tsikoulas, I. (2011). Somatosensory evoked potentials in children with bilateral spastic cerebral palsy. Pediatr. Neurol. 44, 177-182. doi:10.1016/j.pediatrneurol.2010. 11.001

Thomas, B., Eyssen, M., Peeters, R., Molenaers, G., Van Hecke, P., De Cock, P., et al. (2005). Quantitative diffusion tensor imaging in cerebral palsy due to periventricular white matter injury. Brain 128, 2562-2577. doi:10.1093/brain/ awh600

Tomlin, P. I. (1995). The Static Encephalopathies. London: Times-Wolfe International.

Trivedi, R., Agarwal, S., Shah, V., Goyel, P., Paliwal, V. K., Rathore, R. K., et al. (2010). Correlation of quantitative sensorimotor tractography with clinical grade of cerebral palsy. Neuroradiology 52, 759-765. doi:10.1007/s00234-010-0703-8

Van Heest, A. E., House, J., and Putnam, M. (1993). Sensibility deficiencies in the hands of children with spastic hemiplegia. J. Hand Surg. Am. 18, 278-281. doi:10.1016/0363-5023(93)90361-6

Volpe, J. J. (2009). The encephalopathy of prematurity - brain injury and impaired brain development inextricably intertwined. Semin. Pediatr. Neurol. 16, 167-178. doi:10.1016/j.spen.2009.09.005

Wilke, M., and Staudt, M. (2009). Does damage to somatosensory circuits underlie motor impairment in cerebral palsy? Dev. Med. Child Neurol. 51, 686-687. doi:10.1111/j.1469-8749.2009.03332.x
Wingert, J. R., Burton, H., Sinclair, R. J., Brunstrom, J. E., and Damiano, D. L. (2008). Tactile sensory abilities in cerebral palsy: deficits in roughness and object discrimination. Dev. Med. Child Neurol. 50, 832-838. doi:10.1111/j.1469-8749. 2008.03105.x

Wingert, J. R., Burton, H., Sinclair, R. J., Brunstrom, J. E., and Damiano, D. L. (2009). Joint-position sense and kinesthesia in cerebral palsy. Arch. Phys. Med. Rehabil. 90, 447-453. doi:10.1016/j.apmr.2008.08.217

Wingert, J. R., Sinclair, R. J., Dixit, S., Damiano, D. L., and Burton, H. (2010). Somatosensory-evoked cortical activity in spastic diplegic cerebral palsy. Hum. Brain Mapp. 31, 1772-1785. doi:10.1002/hbm.20977

Yeargin-Allsopp, M., Van Naarden Braun, K., Doernberg, N. S., Benedict, R. E., Kirby, R. S., and Durkin, M. S. (2008). Prevalence of cerebral palsy in 8-year-old children in three areas of the United States in 2002: a multisite collaboration. Pediatrics 121, 547-554. doi:10.1542/peds.2007-1270

Yoshida, S., Hayakawa, K., Yamamoto, A., Okano, S., Kanda, T., Yamori, Y., et al. (2010). Quantitative diffusion tensor tractography of the motor and sensory tract in children with cerebral palsy. Dev. Med. Child Neurol. 52, 935-940. doi:10.1111/j.1469-8749.2010.03669.x

Conflict of Interest Statement: The authors declare that the research was conducted in the absence of any commercial or financial relationships that could be construed as a potential conflict of interest.

Received: 14 May 2014; accepted: 28 August 2014; published online: 12 September 2014. Citation: Papadelis C, Ahtam B, Nazarova M, Nimec D, Snyder B, Grant PE and Okada $Y$ (2014) Cortical somatosensory reorganization in children with spastic cerebral palsy: a multimodal neuroimaging study. Front. Hum. Neurosci. 8:725. doi: 10.3389/fnhum.2014.00725

This article was submitted to the journal Frontiers in Human Neuroscience.

Copyright (c) 2014 Papadelis, Ahtam, Nazarova, Nimec, Snyder, Grant and Okada. This is an open-access article distributed under the terms of the Creative Commons Attribution License (CC BY). The use, distribution or reproduction in other forums is permitted, provided the original author(s) or licensor are credited and that the original publication in this journal is cited, in accordance with accepted academic practice. No use, distribution or reproduction is permitted which does not comply with these terms. 